\title{
The Role of Aptamers in the Treatment of Glioblastoma Multiform
}

\section{Sadegh Shirian $^{1 *}$, Reza Khetvan-Hafshejani ${ }^{1 *}$, Ehsan Ali ${ }^{2}$, Sayed Mostafa Modarres Mousavi ${ }^{3,5}$, Sara Abdollahi ${ }^{4,5}$, Nasrin Lotfi Bakhshaiesh ${ }^{6}$}

${ }^{1}$ Department of Pathobiology, School of Veterinary Medicine, Shahrekord University, Shahrekord, Iran

${ }^{2}$ Department of Pharmacology, Qazvin University of Medical Sciences, Qazvin, Iran

${ }^{3}$ Department of Nanobiotechnology, Faculty of Biological Sciences, Tarbiat Modares University, Tehran, Iran

${ }^{4}$ Department of Pathobiology, School of Veterinary Medicine, Shiraz University, Shiraz, Iran

${ }^{5}$ Shefa Neuroscience Research Center, Khatam Alanbia Hospital, Tehran, Iran

${ }^{6}$ Department of Tissue Engineering, Faculty of Advanced Technologies in Medicine, Tehran University of Medical Sciences, Tehran, Iran

\section{Article Info:}

\section{A BSTRACT}

Introduction: Glioblastoma multiform (GBM) or grade IV glioma is the most common and aggressive primary human brain tumor. Primary GBMs which arise spontaneously from glial cells and represent the vast majority of GBM are usually related to epidermal growth factor receptor amplification and mutations, deletion of phosphatase and tensin homolog (PTEN) on chromosome 10, p16 deletion, and TERT promoter mutation. Despite advances in treatment over the past few decades the median survival time of GBM patients is 12.1-14.6 months after diagnosis and only $3-5 \%$ of patients survive longer than 3 years. The effect of usual treatments, including surgery, radiotherapy, chemotherapy, and a combination of radiotherapy and chemotherapy, have extremely low effectiveness. Although surgery and temozolomide application during and after radiotherapy increases patient survival time, they have various side effects. Therefore, introducing a novel drug that able to inhibit GBM cell growth, proliferation, migration, and invasion is required. Since cancer cells have surface molecules that are over-expressed or altered, the molecular structures called aptamers have been designed using systematic evolution of ligands by exponential enrichment (SELEX) process to target these surface molecules. To date, several aptamers with advantages and disadvantages have been applied for the treatment of different diseases, including cancer, that are discussed in this study. Conclusion: Several aptamers with high capacity in the detection of GBM have been recently designed using the SELEX process that shows the high potential of aptamers in the management of GBM.

\section{Key words:}

1. Glioblastoma

2. Aptamers, Nucleotide

3. Oligodendroglia

*Corresponding Author: Sadegh Shirian, Reza Khetvan-Hafshejani

E-mail:Shiran85@gmail.com,rezakhetvan@gmail.com 


\section{نقش آيتامرها در درمان كليوبلاستوم مولتىفرم}

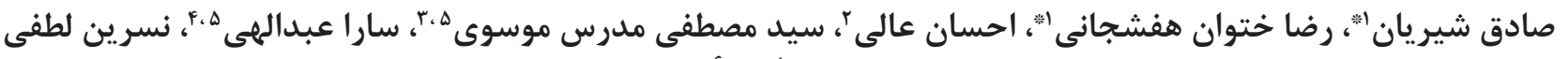 بخشايش

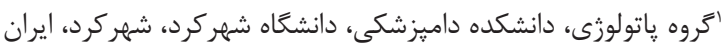

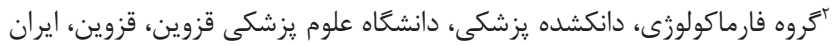

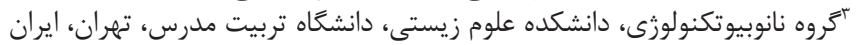

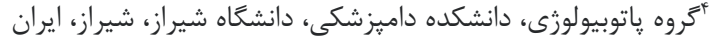

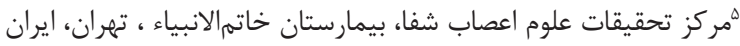

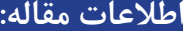

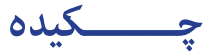

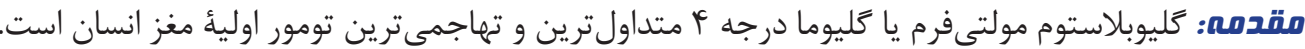

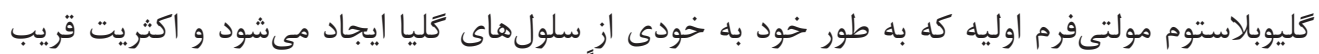

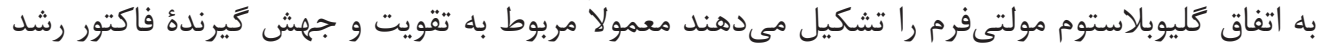

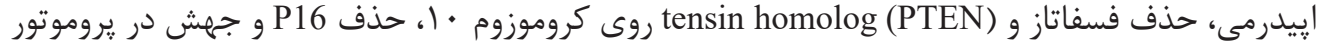

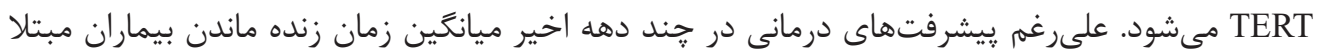

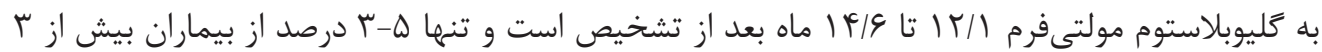

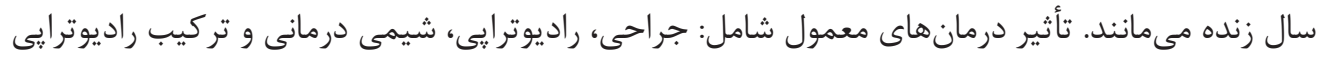

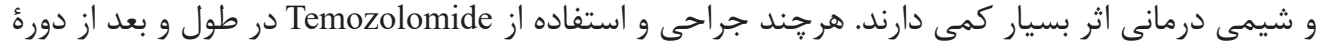

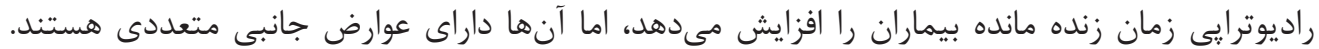

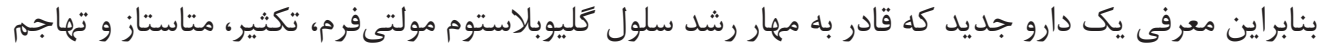

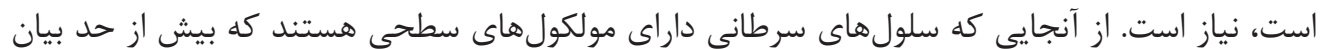

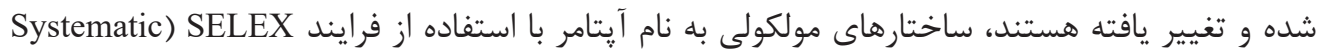
(Evolution of Ligands by Exponential enrichment

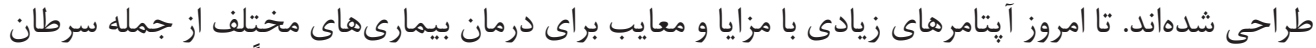

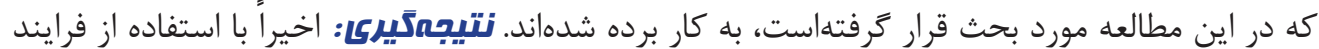
SELEX

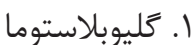

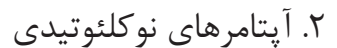

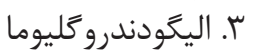

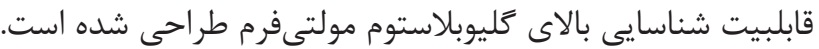




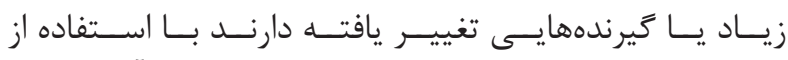

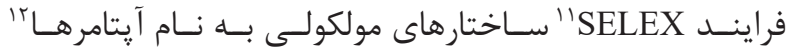

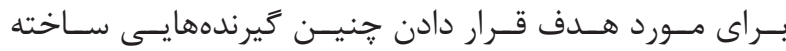

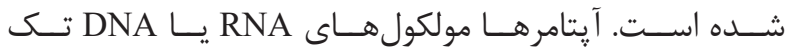

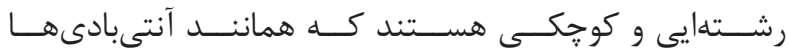

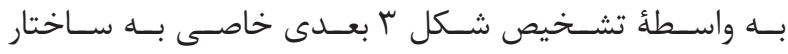

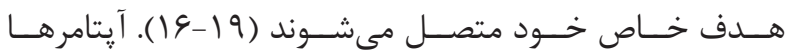

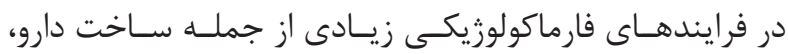

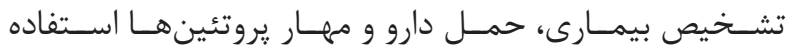

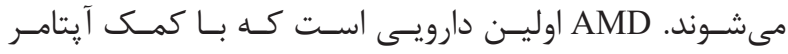

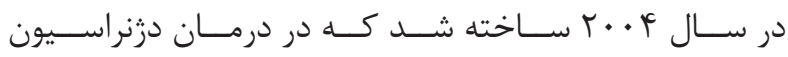

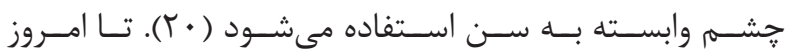

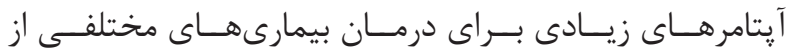

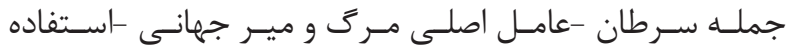

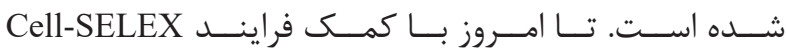

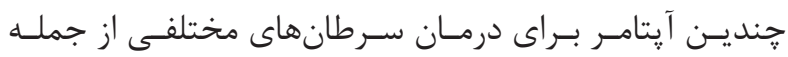

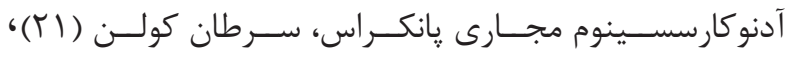

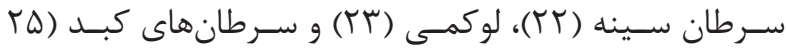

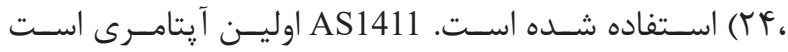

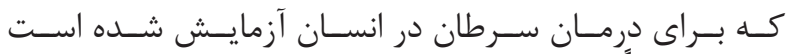

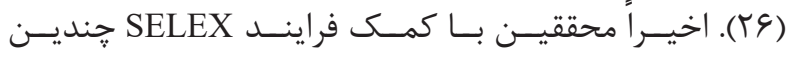

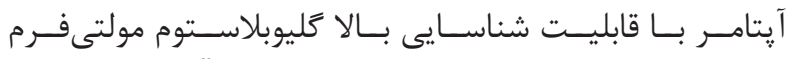

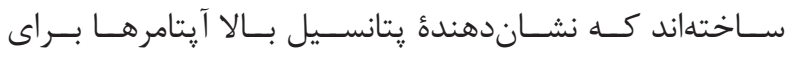

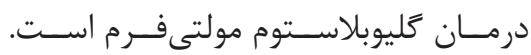

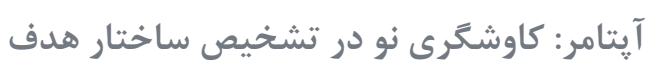

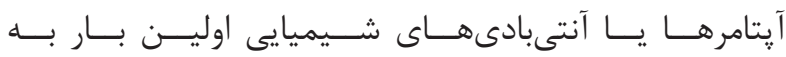

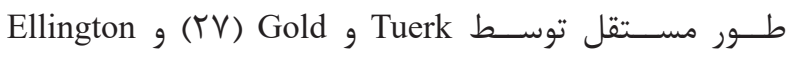

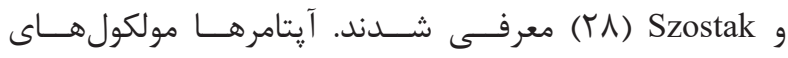

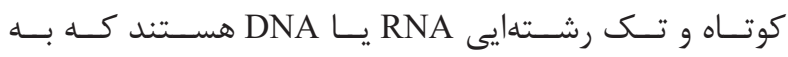

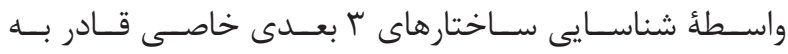

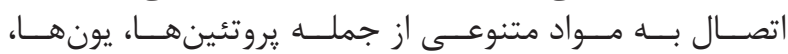

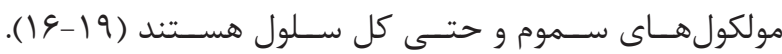

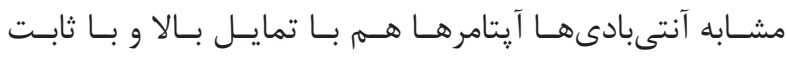

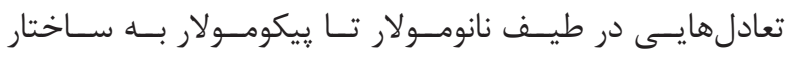

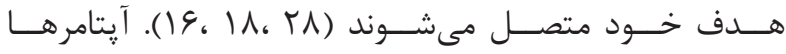

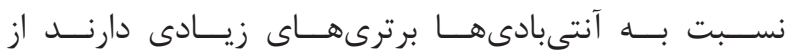

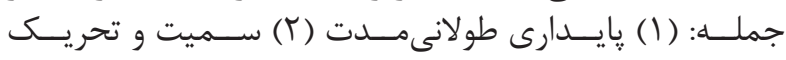

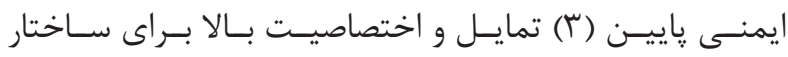

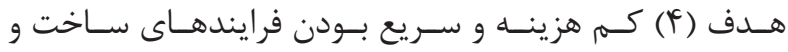

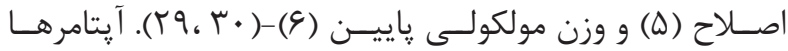

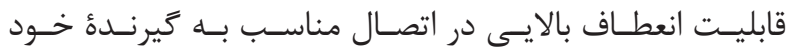

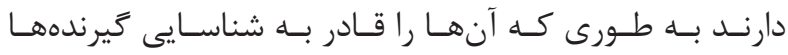

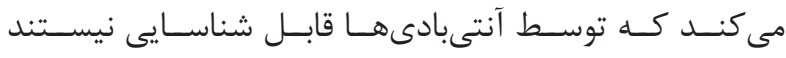

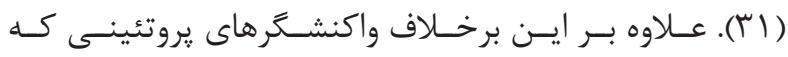

${ }^{1}$ Gliobastoma multiforme

${ }^{2}$ Glioma

${ }^{3}$ Primary

${ }^{4}$ Epidermal growth factor receptor

${ }^{5}$ Phosphatase and tensin homolog

${ }^{6}$ Secondary

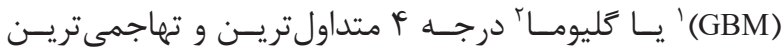

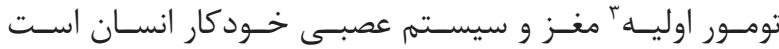

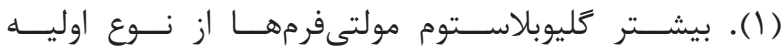

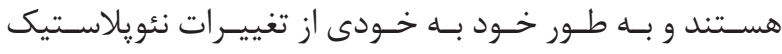

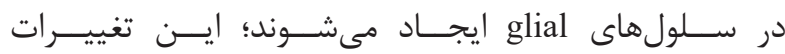

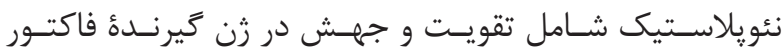

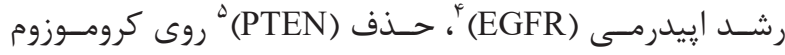

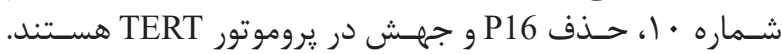

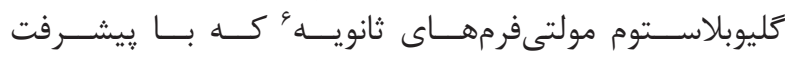

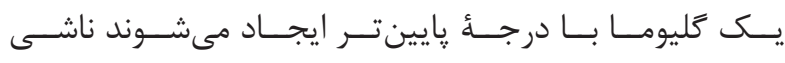

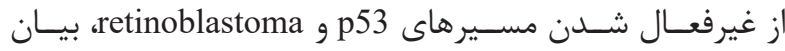

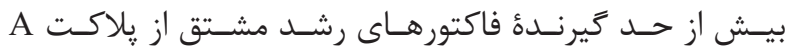

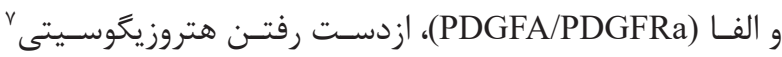

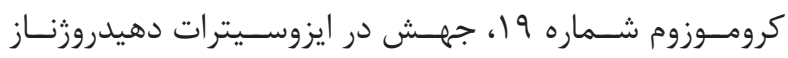

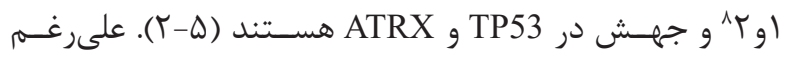

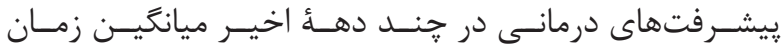

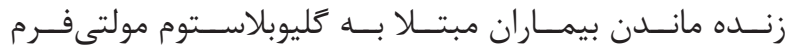

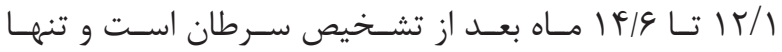

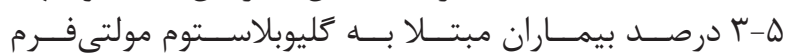

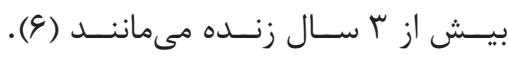

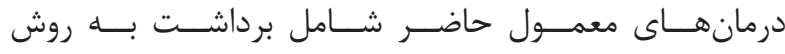

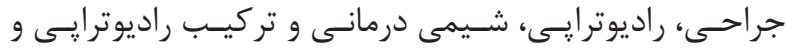

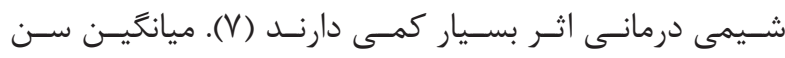

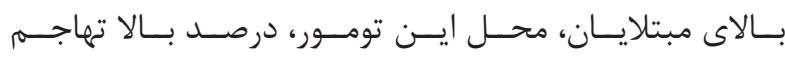

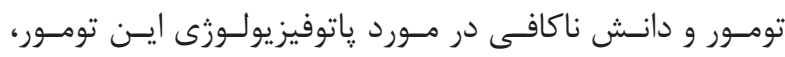

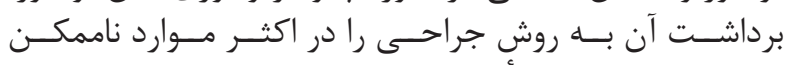

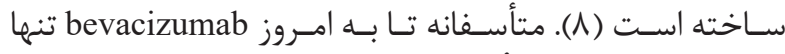

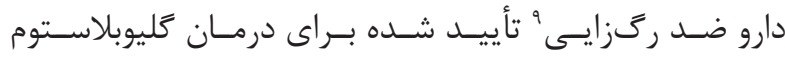

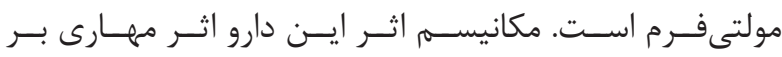

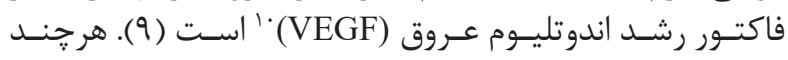

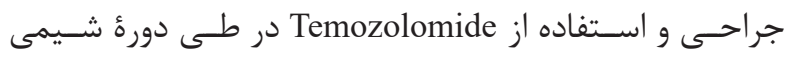

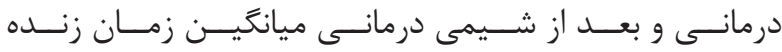

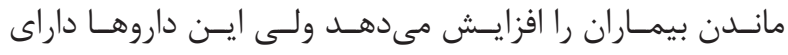

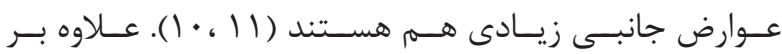

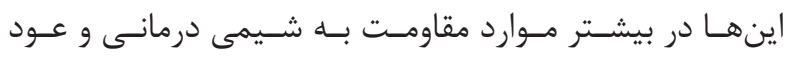

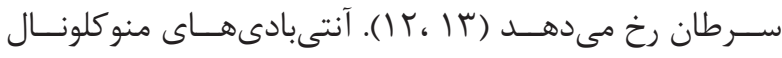

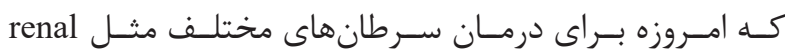

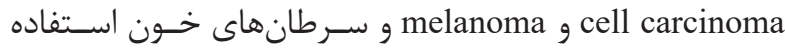

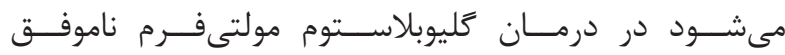

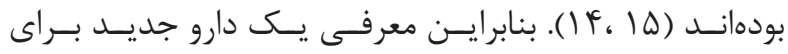

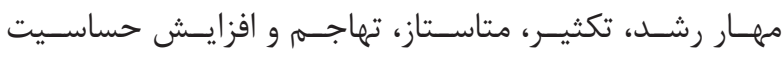

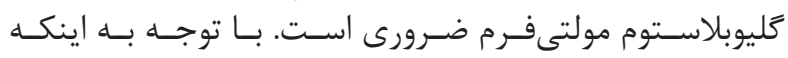

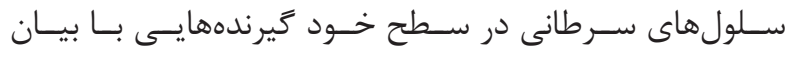

\footnotetext{
${ }^{7}$ Heterozygosity

${ }^{8}$ Isocitrate dehydrogenase

${ }^{9}$ Angiogenesis

${ }^{10}$ Vascular endothelial growth factor

${ }^{11}$ Systematic evolution of ligands by exponential enrichment

${ }^{12}$ Aptamers
} 


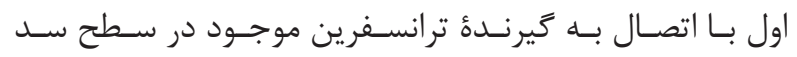

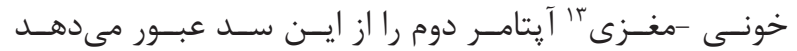

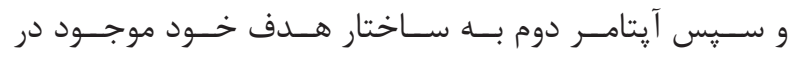

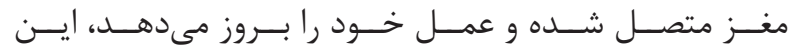

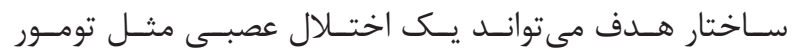

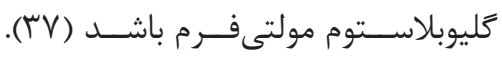

آيتامر هايى با ساختار هدفى به نام Nucleolin

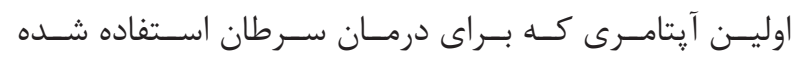

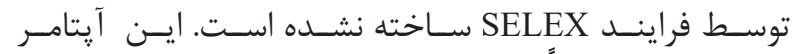

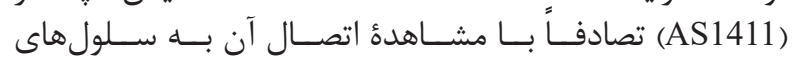

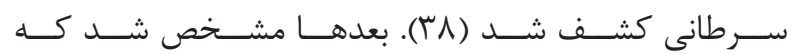

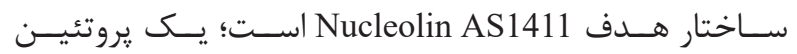

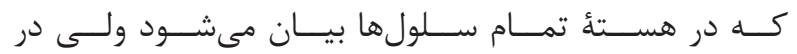

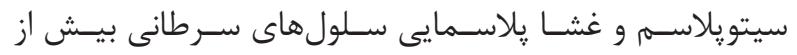

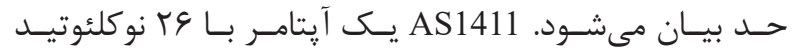

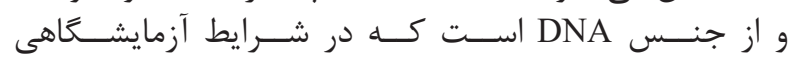

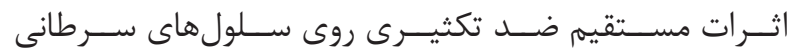

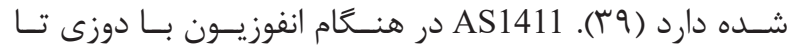

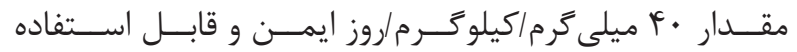

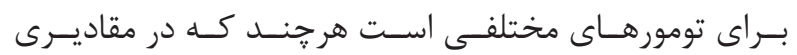

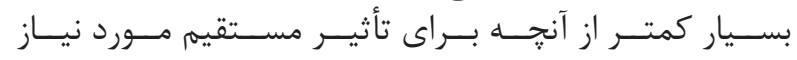

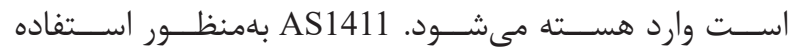

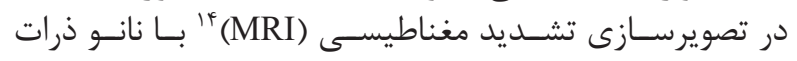

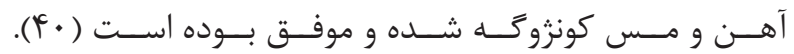

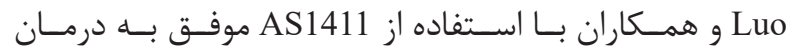

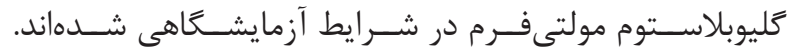

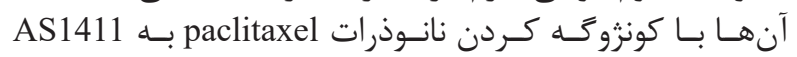

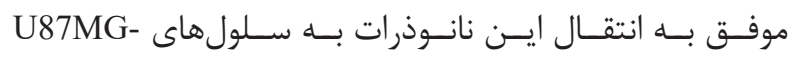

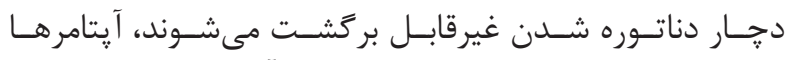

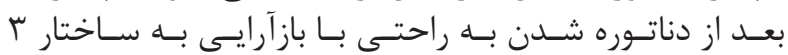

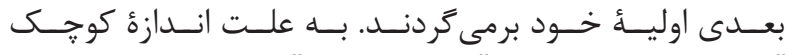

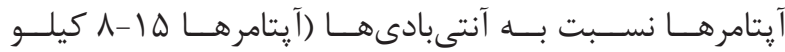

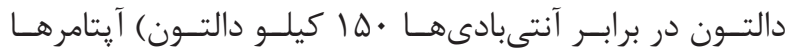

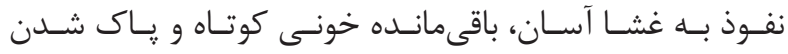

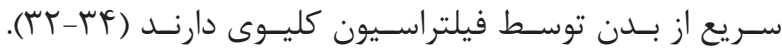

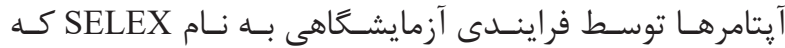

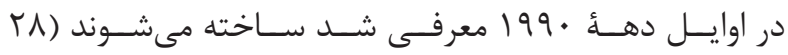

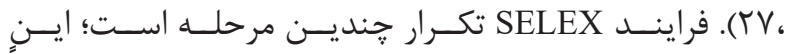

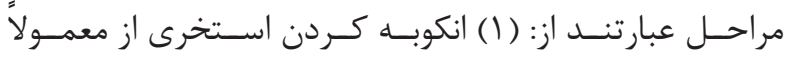

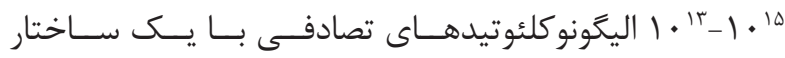

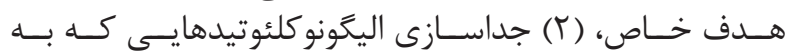

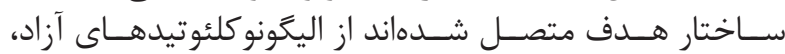

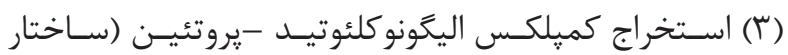

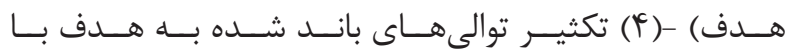

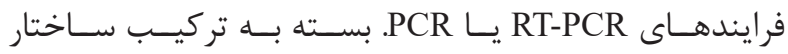

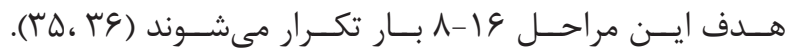

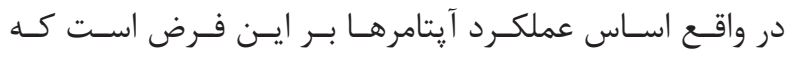

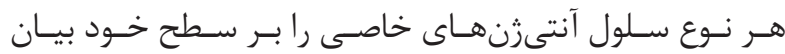

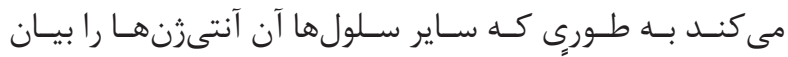

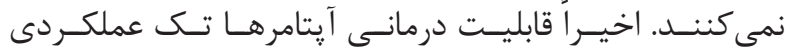

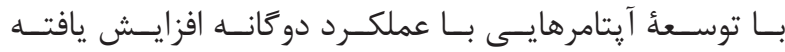

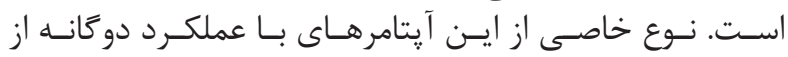

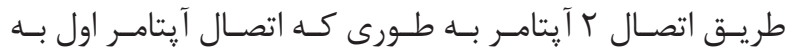

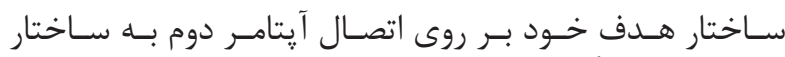

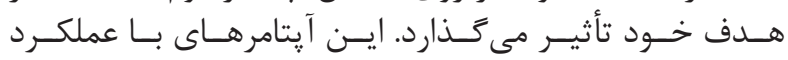

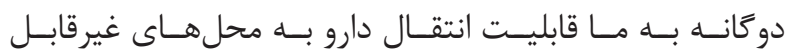

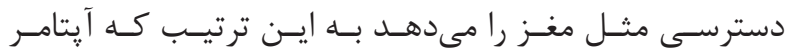

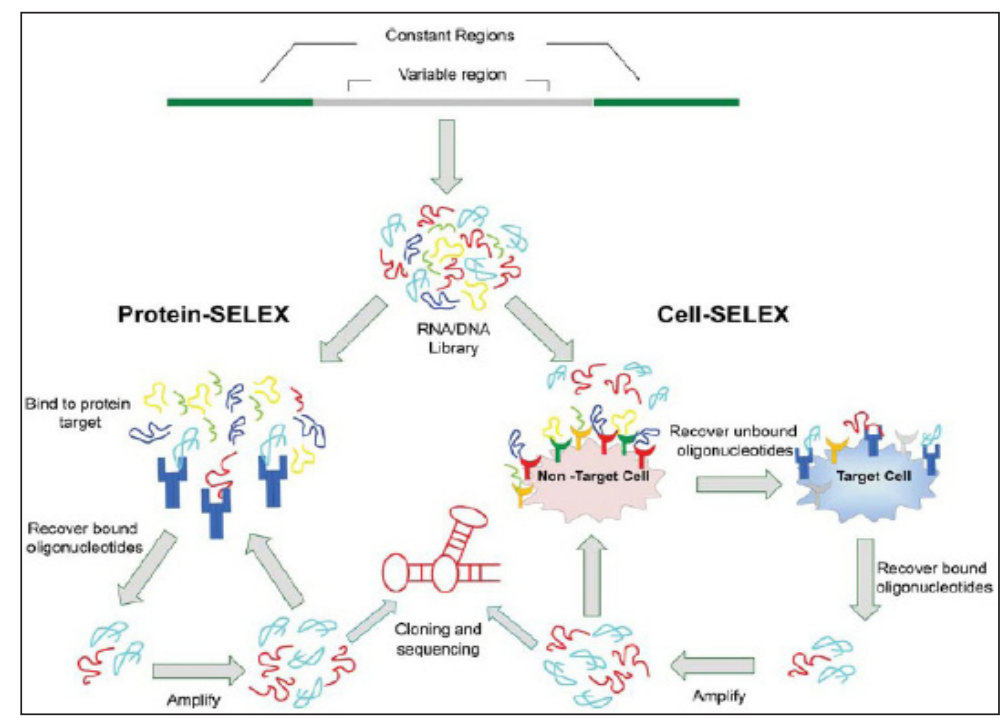

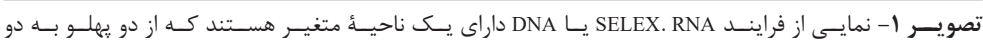

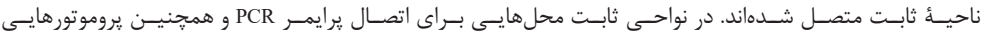

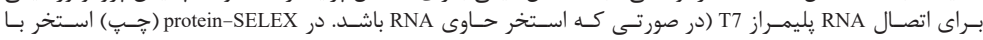

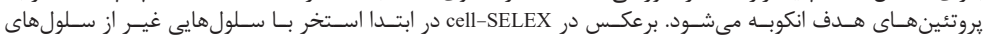

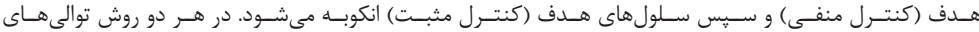

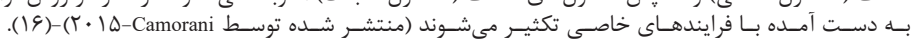

${ }^{13}$ Blood brain barrier

${ }^{14}$ Magnetic resonance imaging 


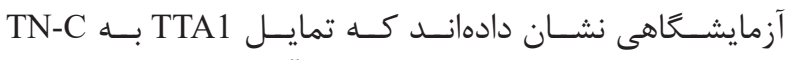

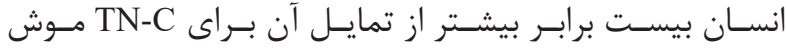

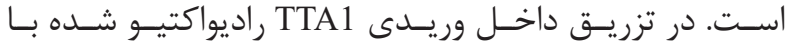

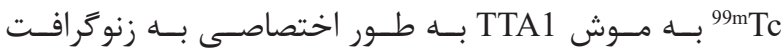

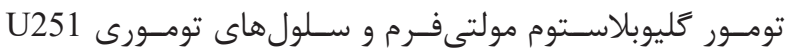

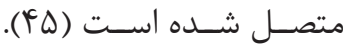

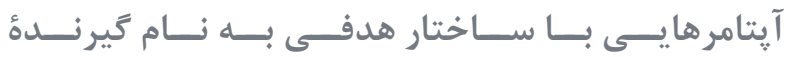

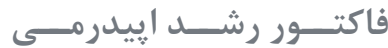

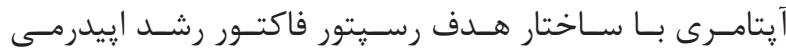

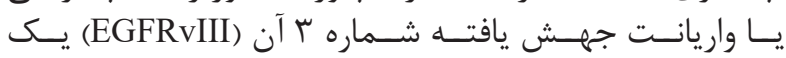

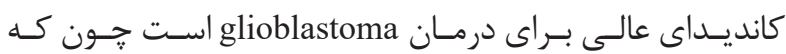

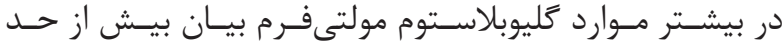

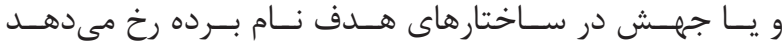

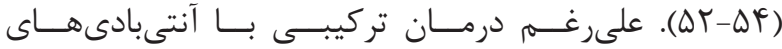

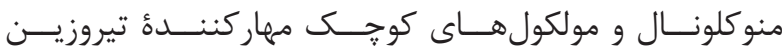

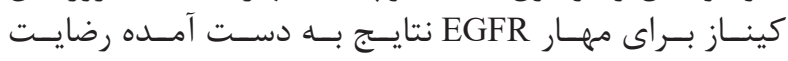

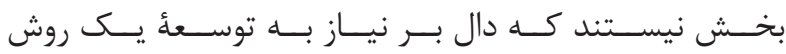

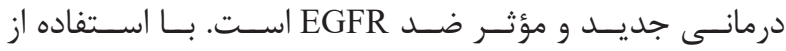

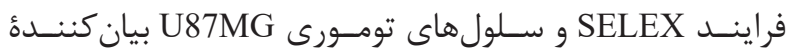

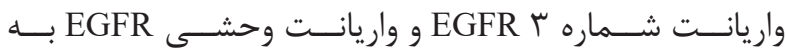

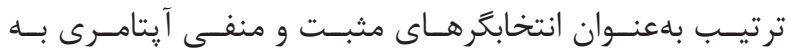

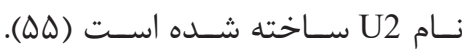

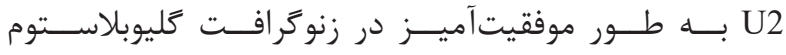

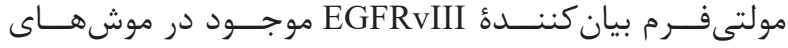

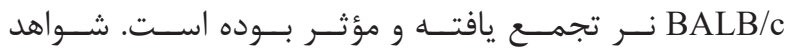

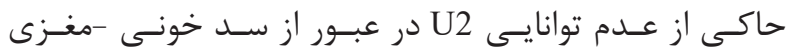

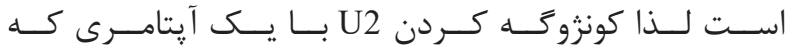

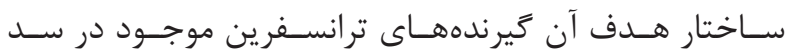

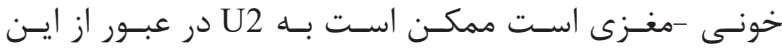

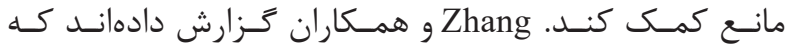

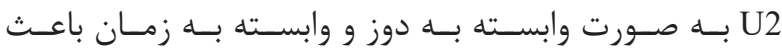

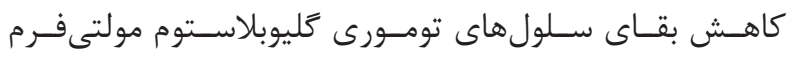

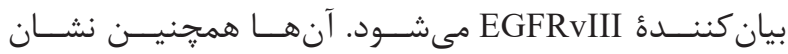

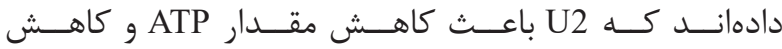

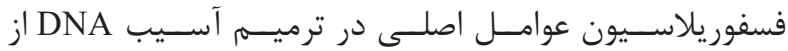

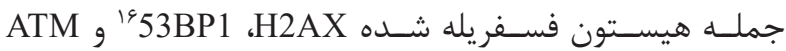

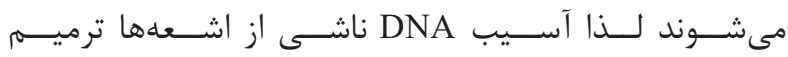

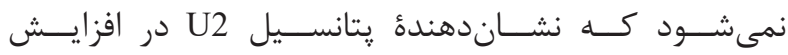

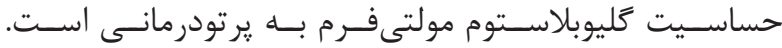

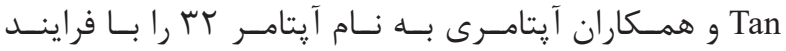

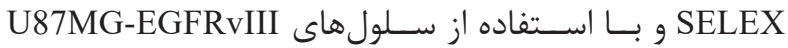

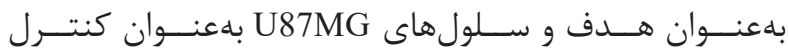

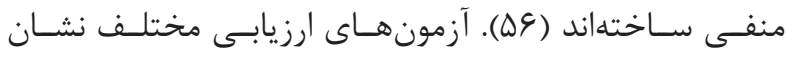

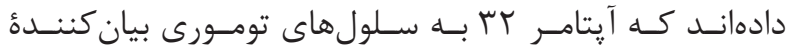
(U87MG-EGFRvIII) EGFRvIII

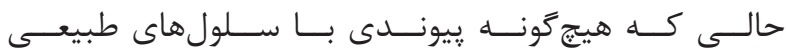

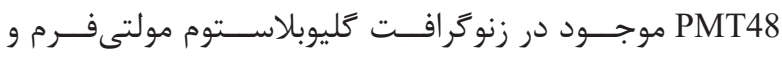

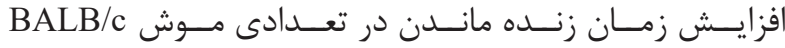

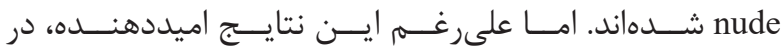

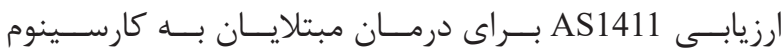

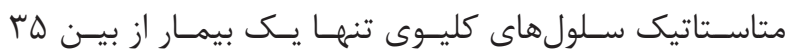

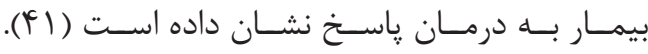
آيتامر هايى با ساختار هدفى به نام Tenascin-C (TN-C)

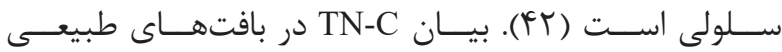

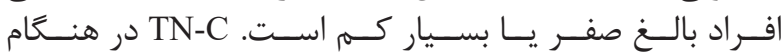

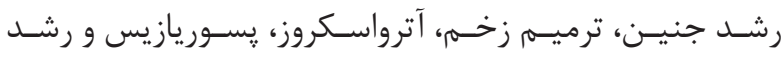

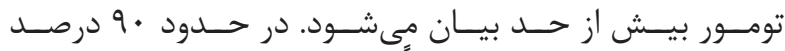

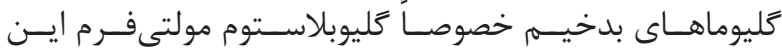

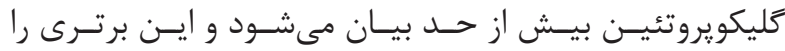

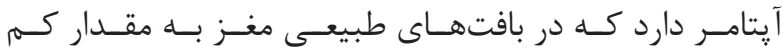

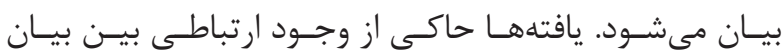

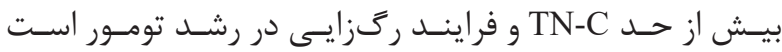

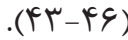

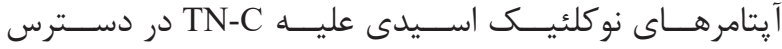

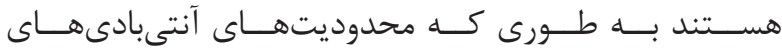

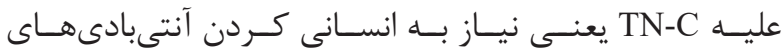

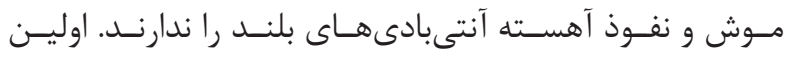

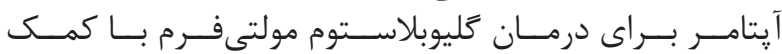

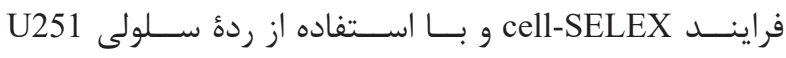

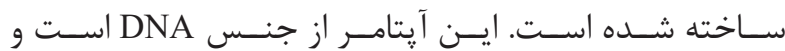

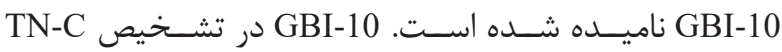

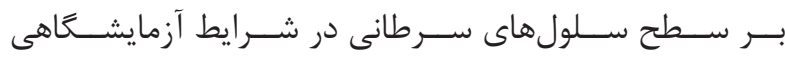

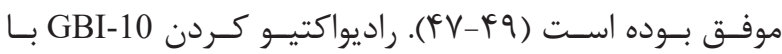

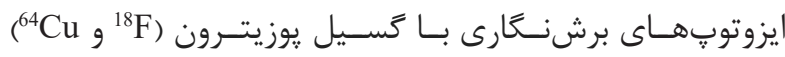

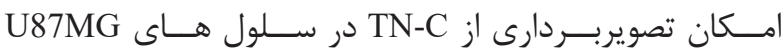

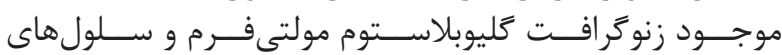

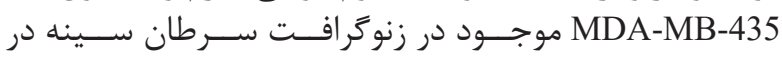

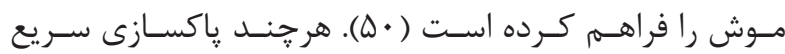

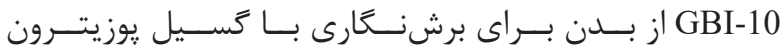

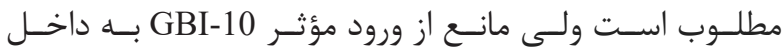

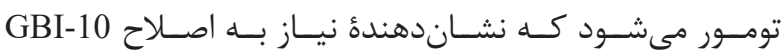

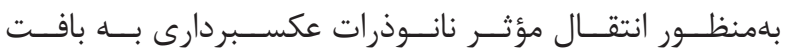

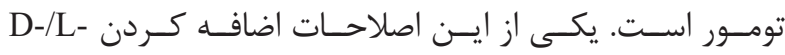
GBI-10 20-dI phosphoramidite و isonucleotides

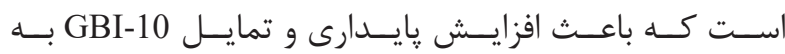

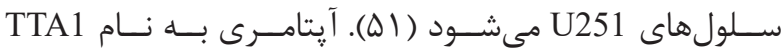

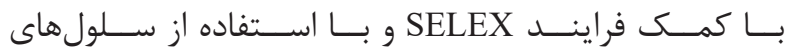

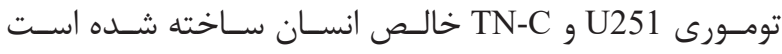

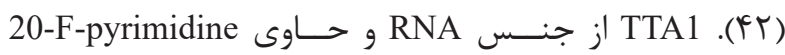

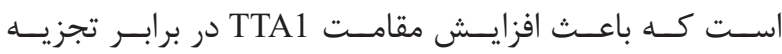

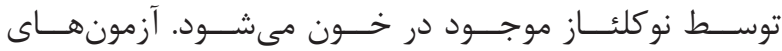


ورود ســلول هاى اســترومال بــهـ ريزمحيـط اطــــاف تومــور،

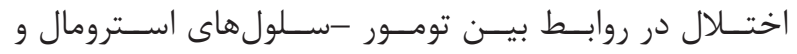

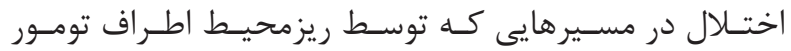

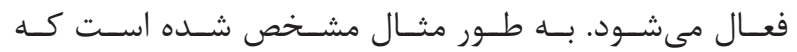

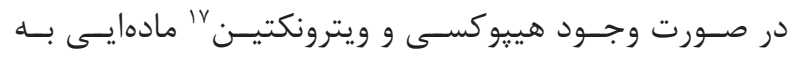

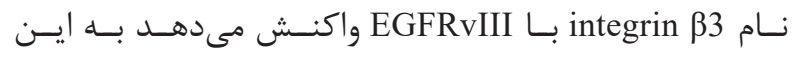

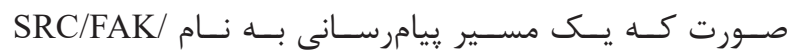
EGFRvIII

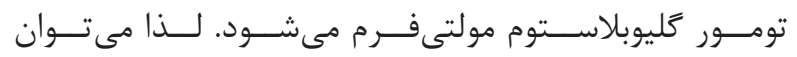

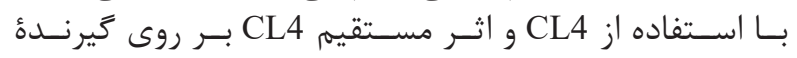
EGFRvIII

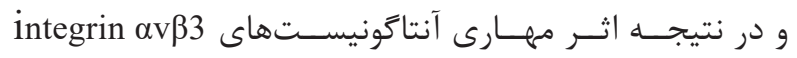

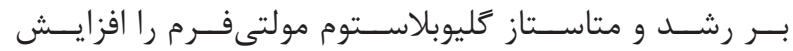

داد (GF)

آيتامر هايى با ساختار هدف Axl

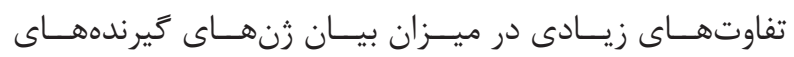

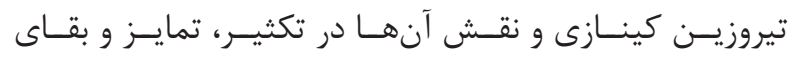

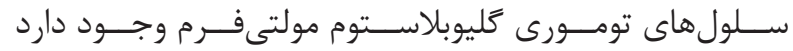

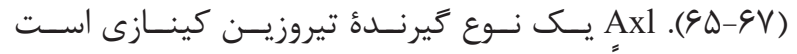

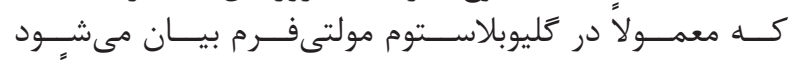

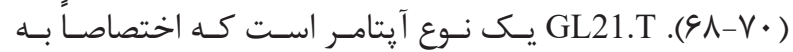

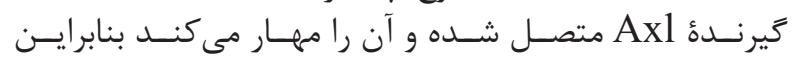

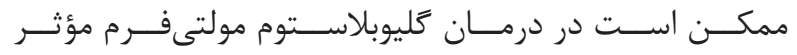

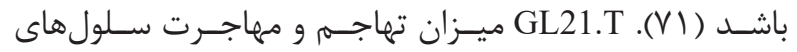

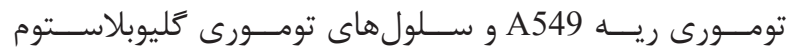

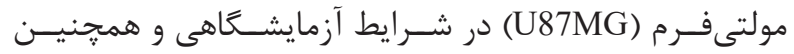

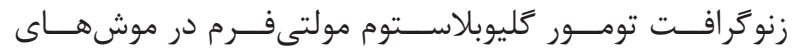

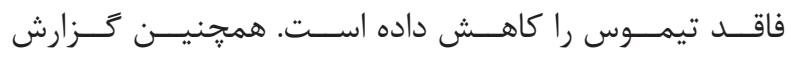

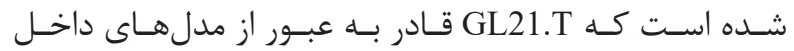

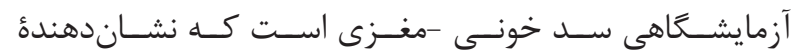

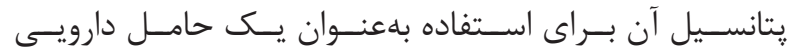
در كلينيـك اسـت (VT)

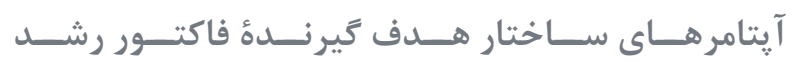

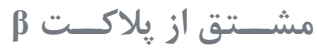

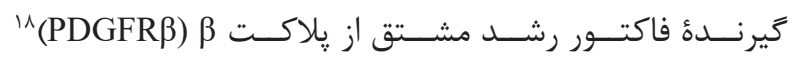

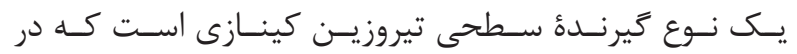

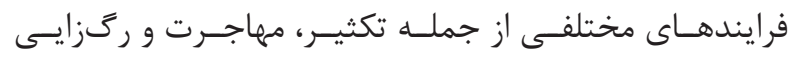

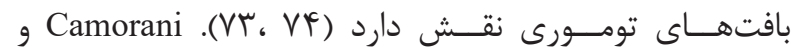

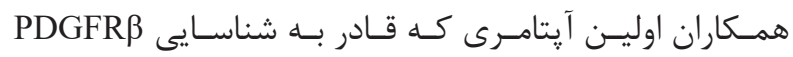

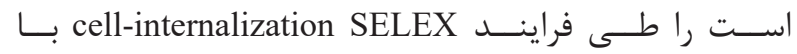

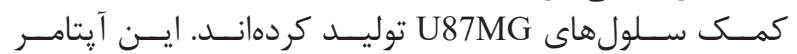
(Gint4.T)

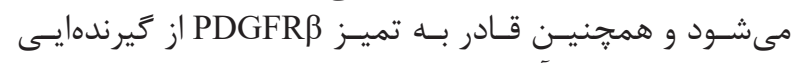

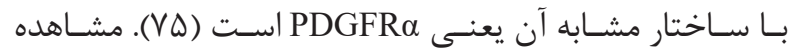

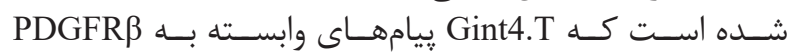

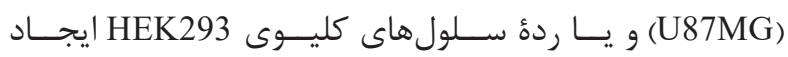

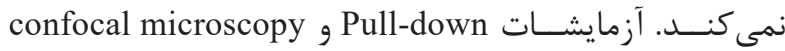

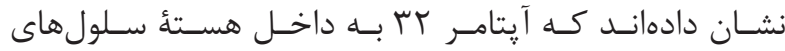

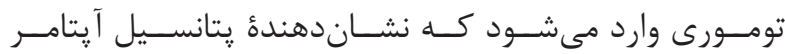

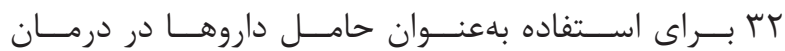

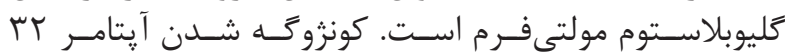

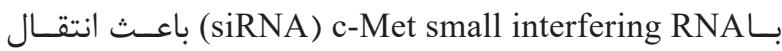
موفـ SiRNA بـه سـلول هاى هـدف (U87MG-EGFRvIII)

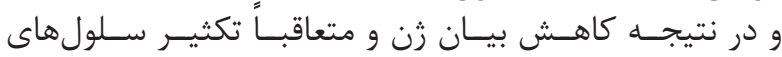

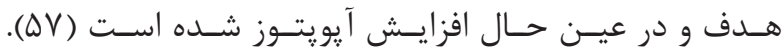

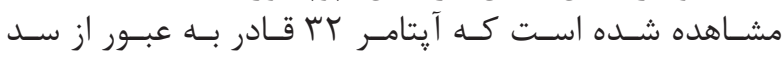

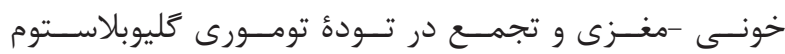

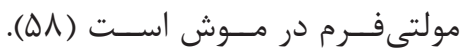

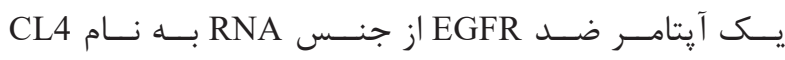

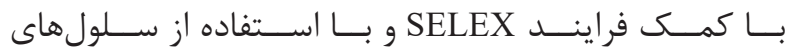

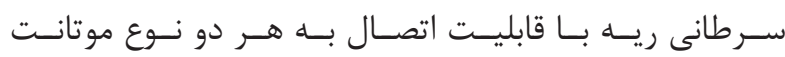

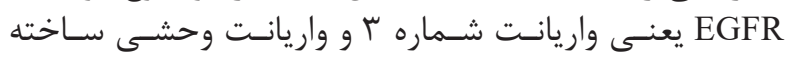

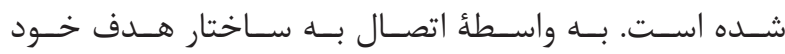

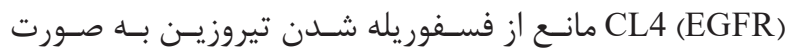

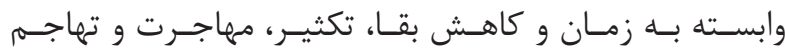

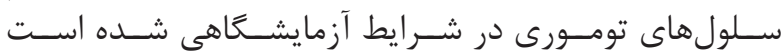

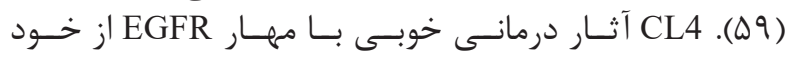

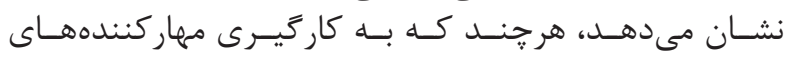

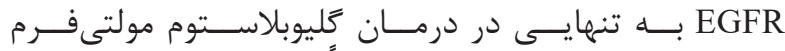

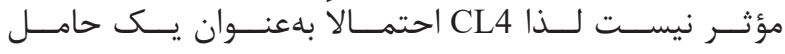

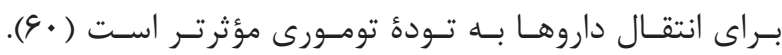

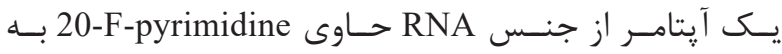

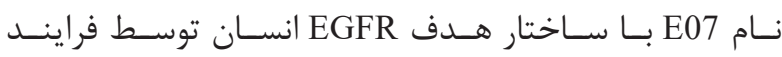
SELEX

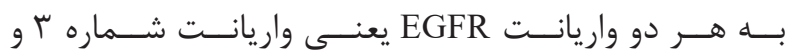

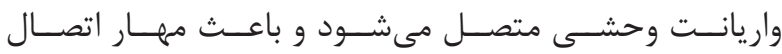

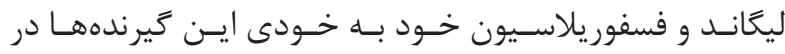

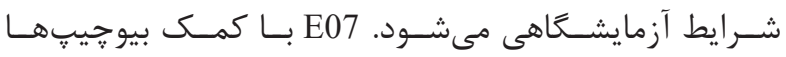

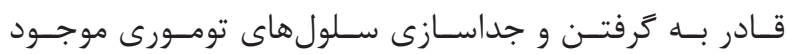

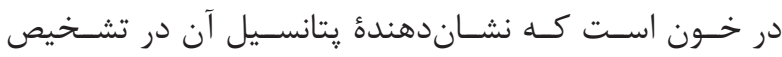

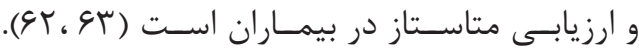

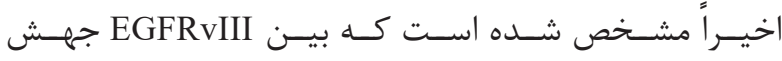

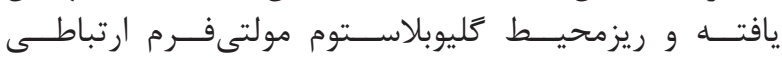

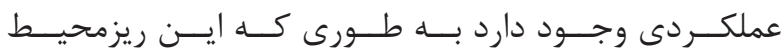

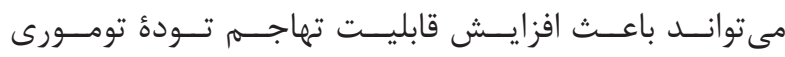

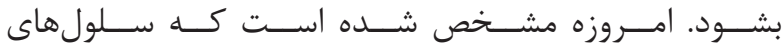

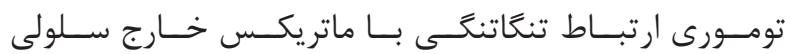

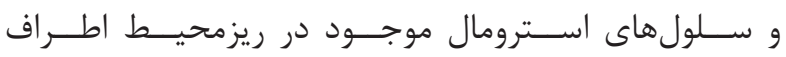

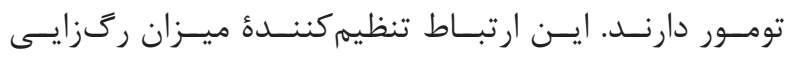

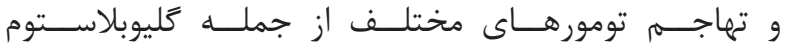

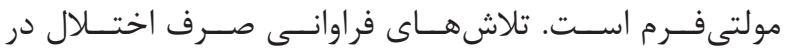

${ }^{17}$ Vitronectin

${ }^{18}$ Platelet derived growth factor receptor-beta 


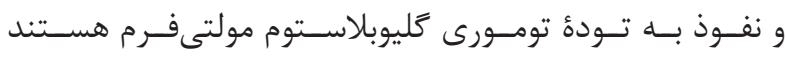

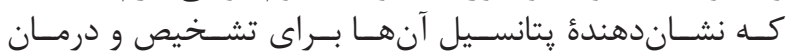

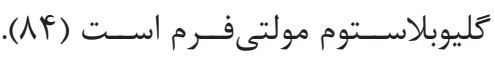

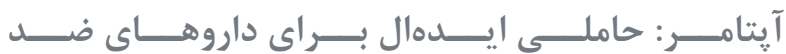

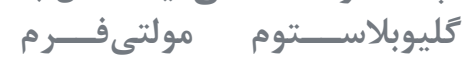

كزارشهــاى زيــادى مبنــى بـــر توانايسى آيتامرهــا در عبــور

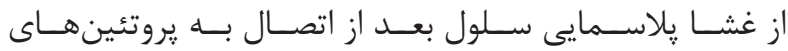

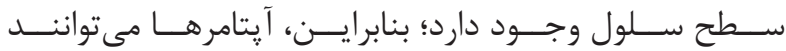

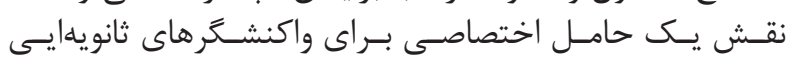

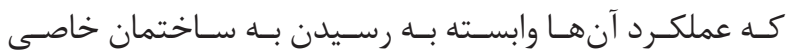

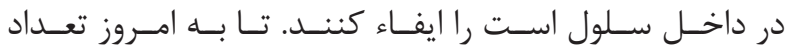

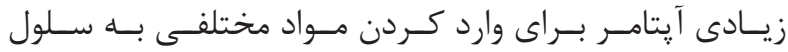

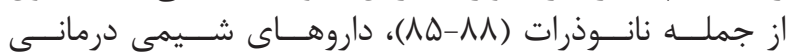

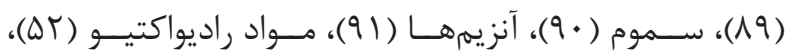
-qV) ${ }^{r}$ (miRNAs) و (qr، qr) small interfering RNAs

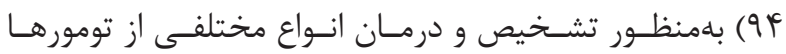

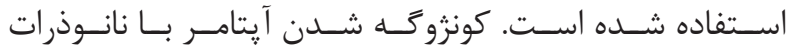

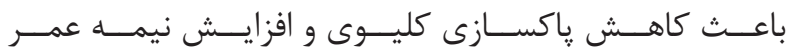

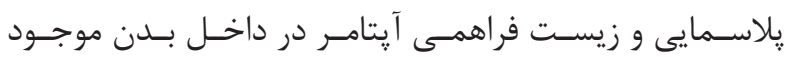

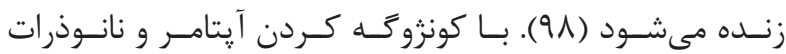

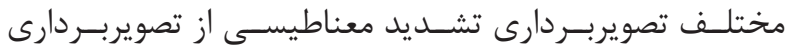

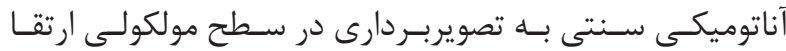

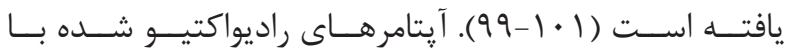

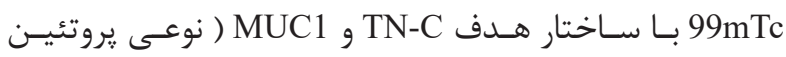

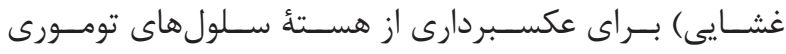

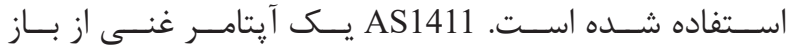

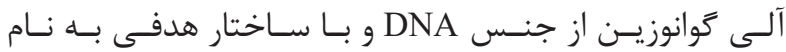

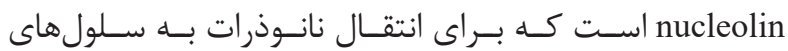

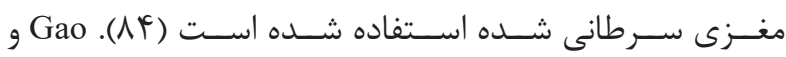

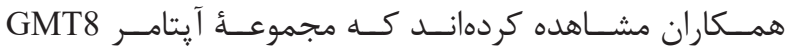

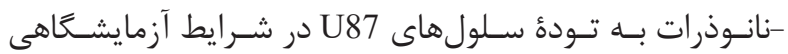

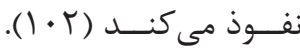

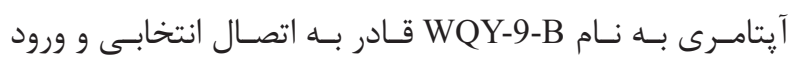

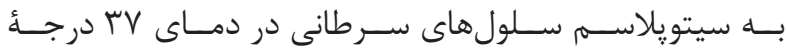

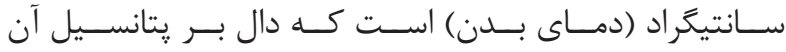

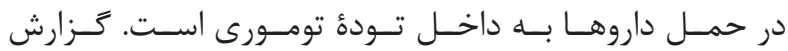

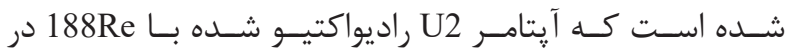

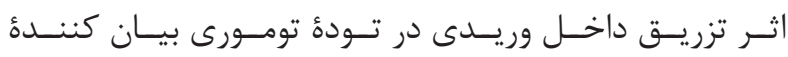
EGFRvIII

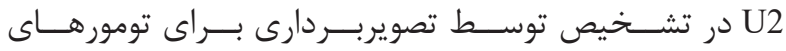

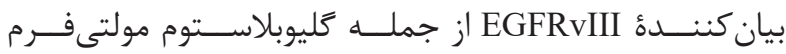

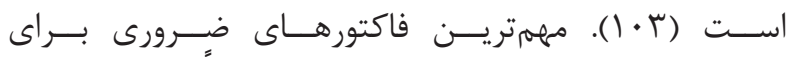

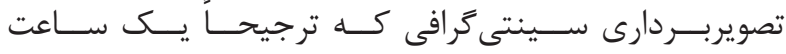

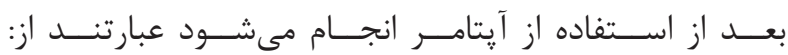

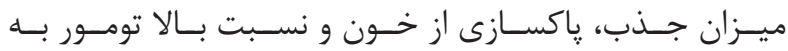

${ }^{19}$ GBM stem cells

${ }^{20}$ Tumor-initiating cells

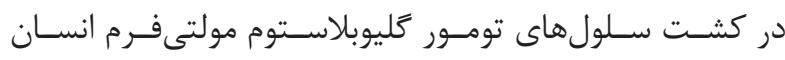

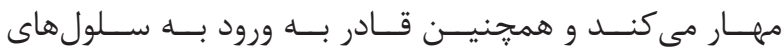

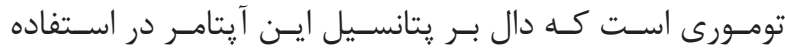

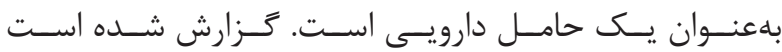

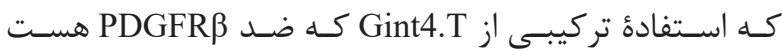

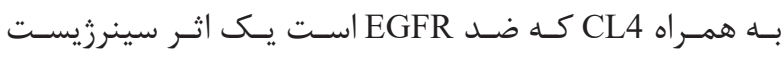

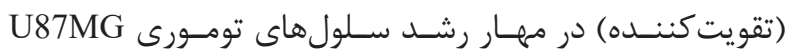

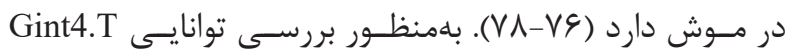

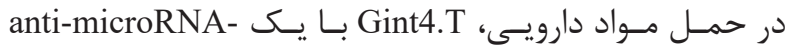

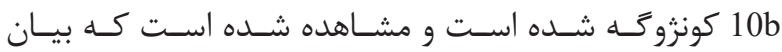

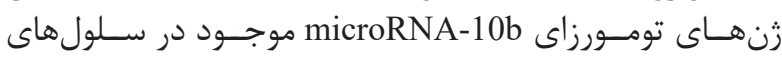

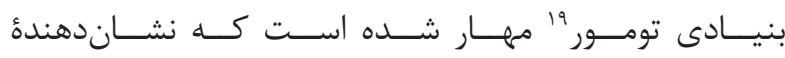

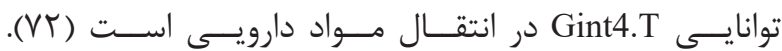

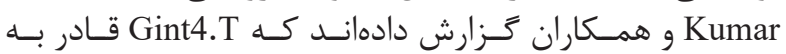

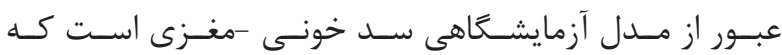

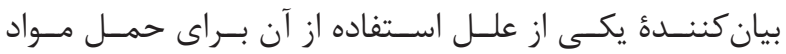

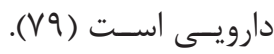

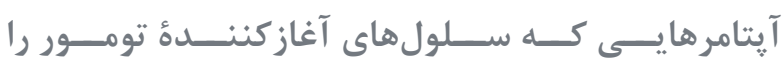

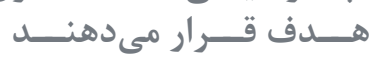

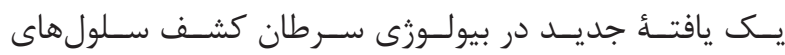

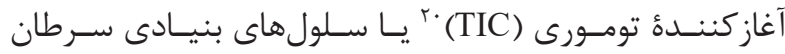

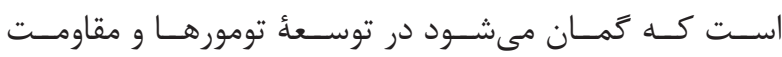

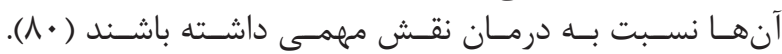

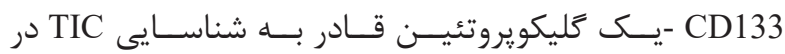

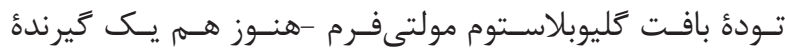

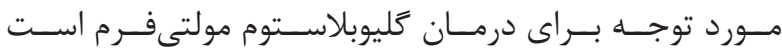

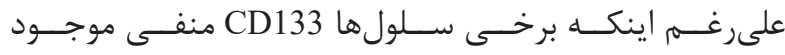

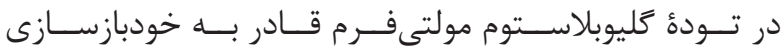

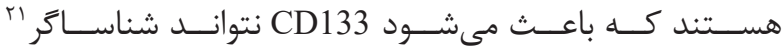

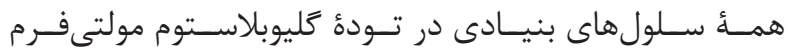

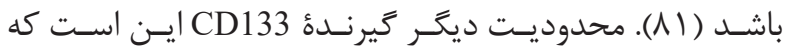

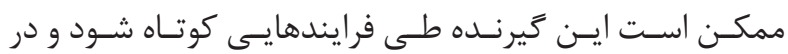

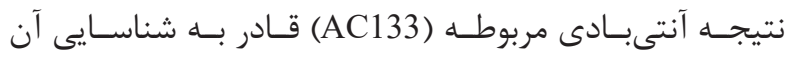

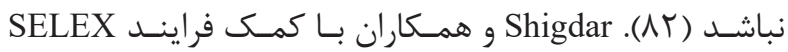

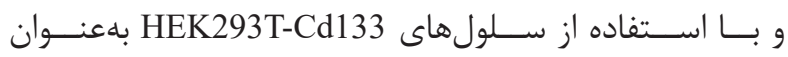

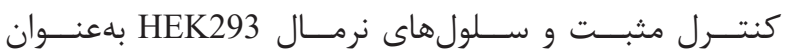

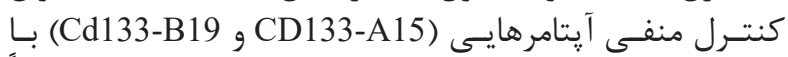

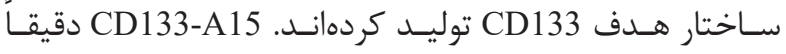

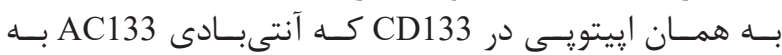

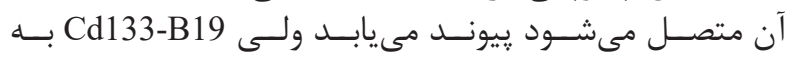

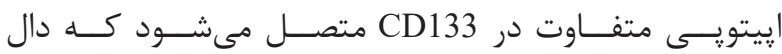

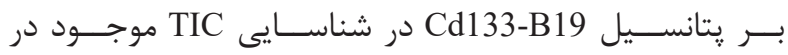

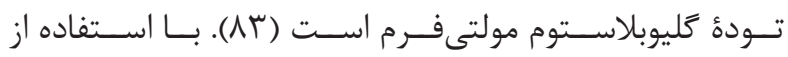

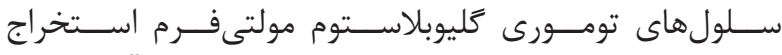

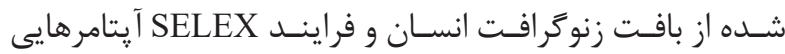

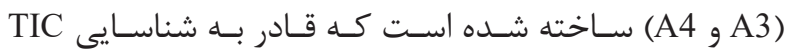

\footnotetext{
${ }^{21}$ Marker

${ }^{22}$ MicroRNAs
} 


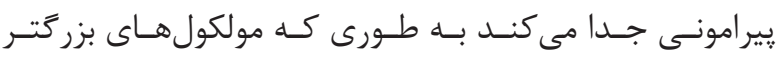

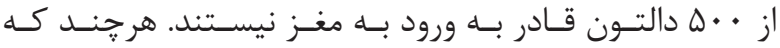

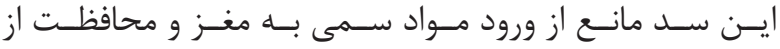

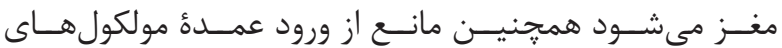

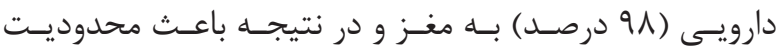

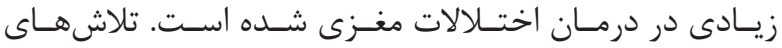

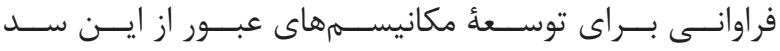

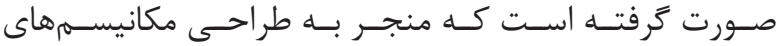

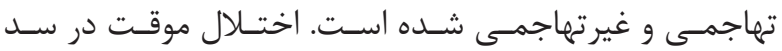

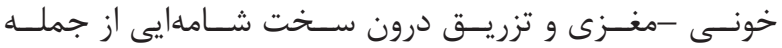

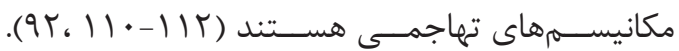

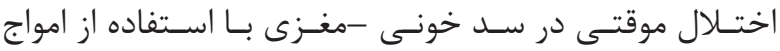

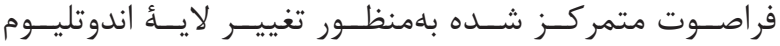

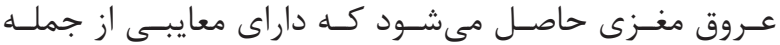

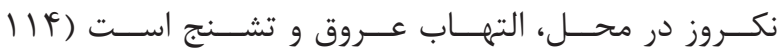

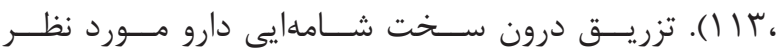

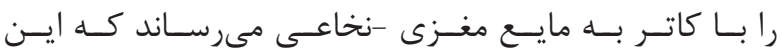

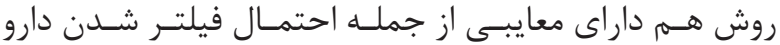

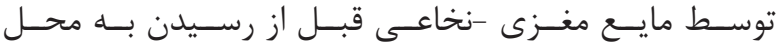

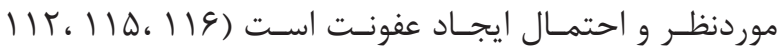

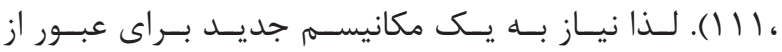

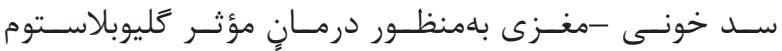

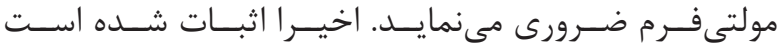

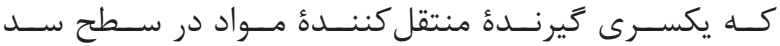

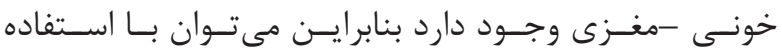

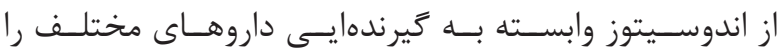

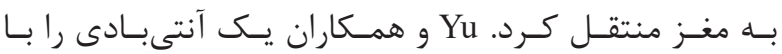

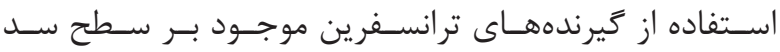

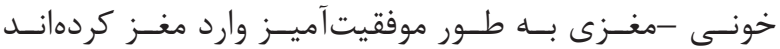
(IVV)

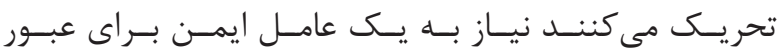

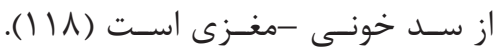

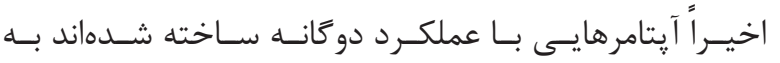

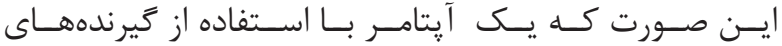

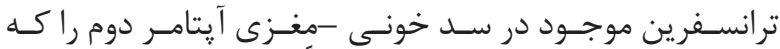

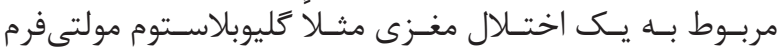

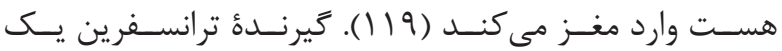

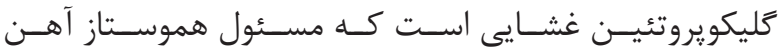

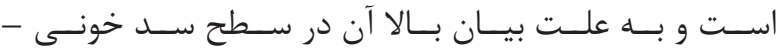

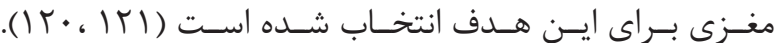

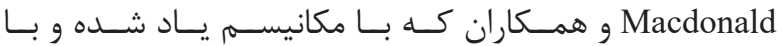

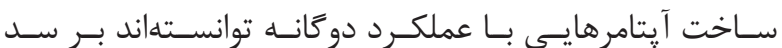

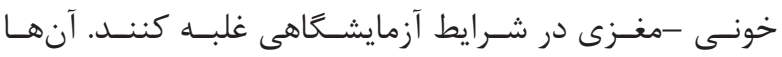

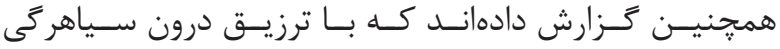

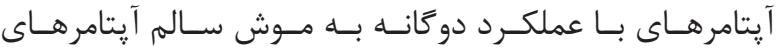

${ }^{23}$ Polyethylene glycol

${ }^{24}$ Diethylene-triamine-penta-acetic acid

${ }^{25}$ Viral therapy delivery system

${ }^{26}$ Constitutive androstane receptor

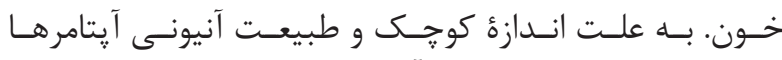

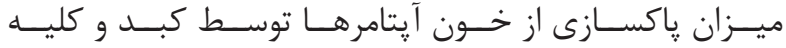

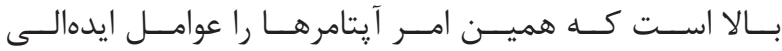

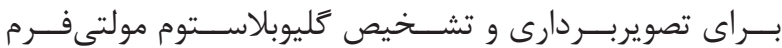

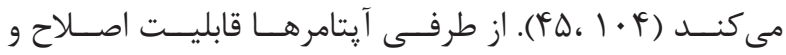

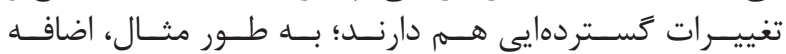
كــردن (PEG)

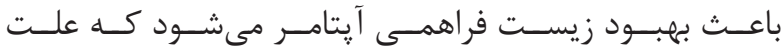

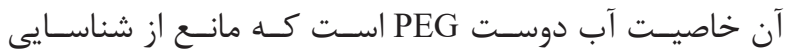

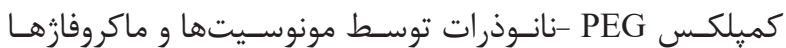

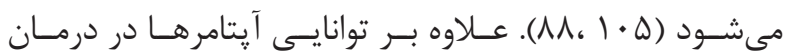

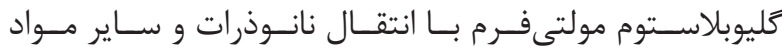

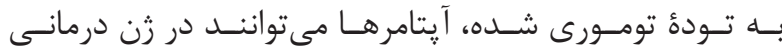

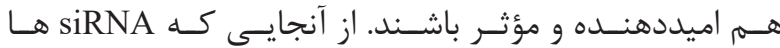

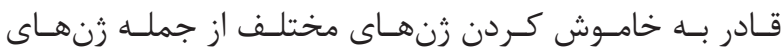

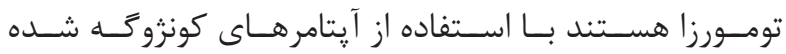

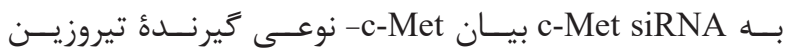

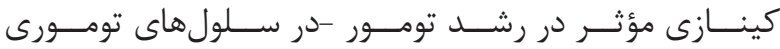
U87-EGFRvIII

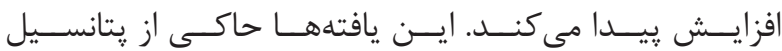

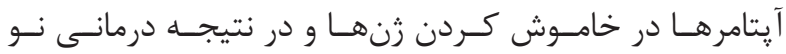

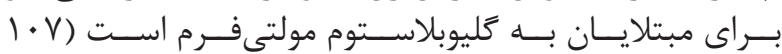

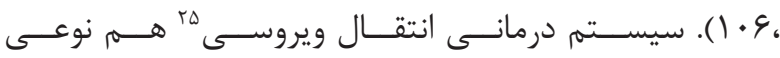

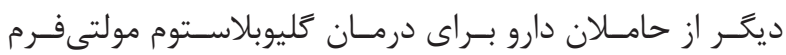

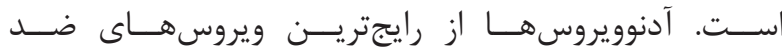

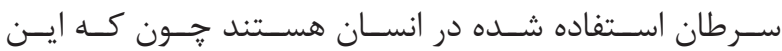

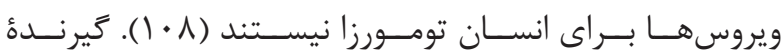

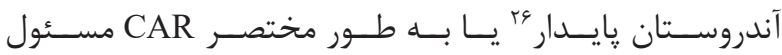

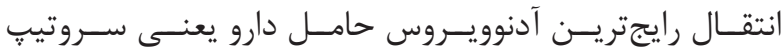

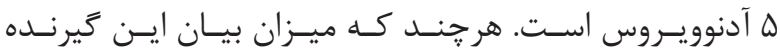

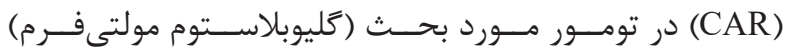

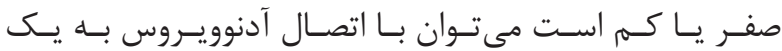

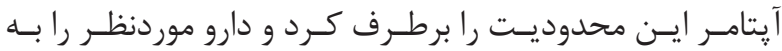

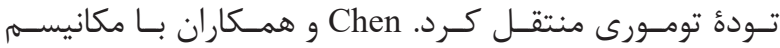

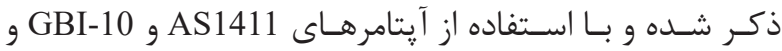

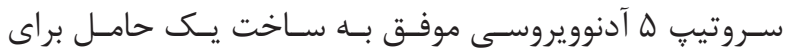

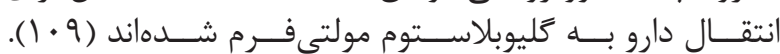

$$
\text { غلبه بر سد خونى -مغزى }
$$

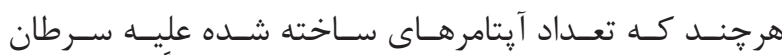

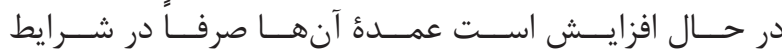

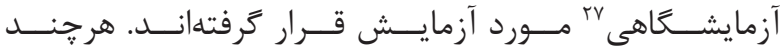

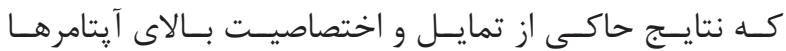

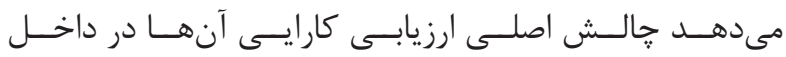

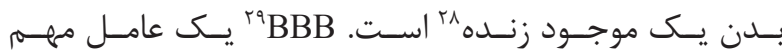

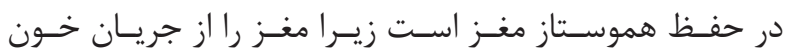

\footnotetext{
${ }^{27}$ In vitro

${ }^{28}$ In vivo

${ }^{29}$ Blood - brain barrier
} 


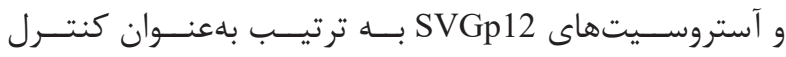

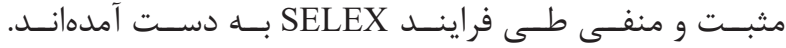

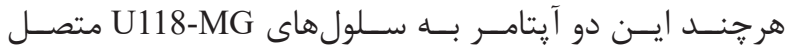

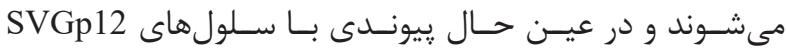

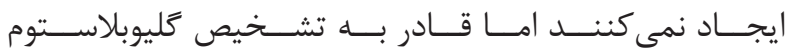

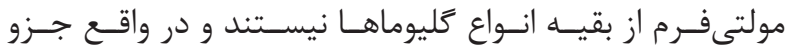

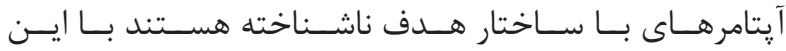

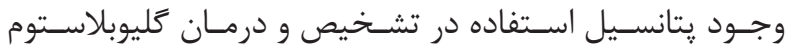

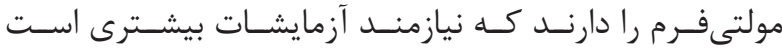

(IT)

نتيجه گَيرى

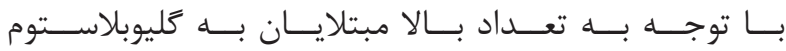

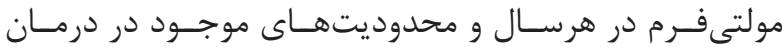

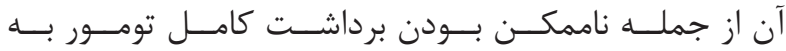

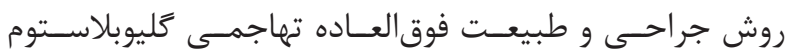

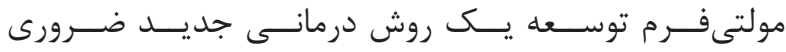

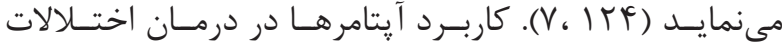

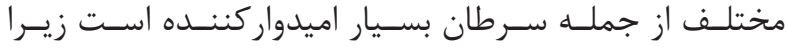

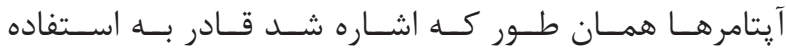

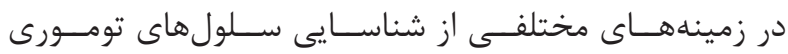

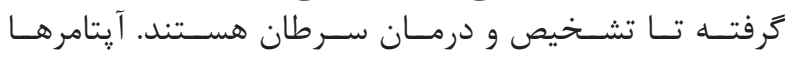

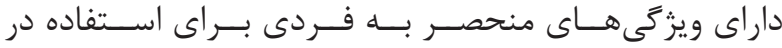

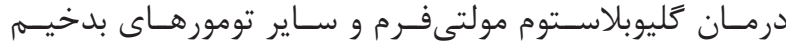

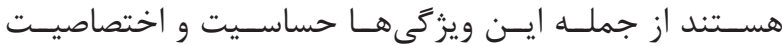

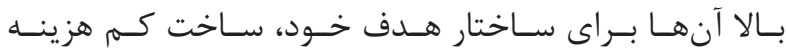

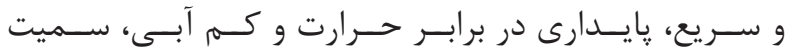

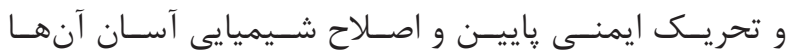

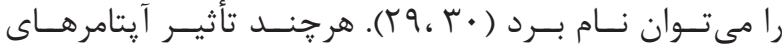

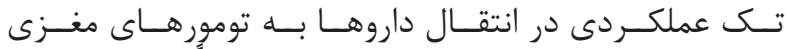

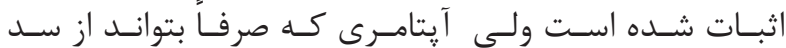

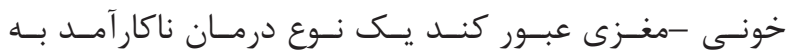

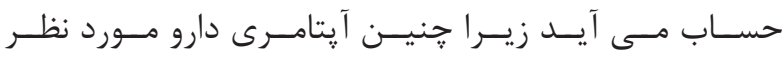

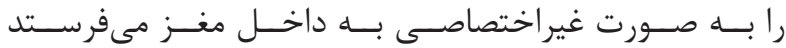

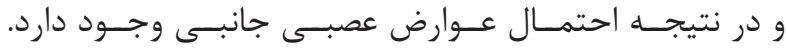

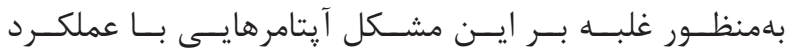

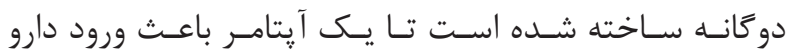

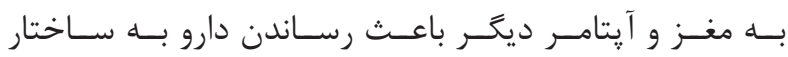

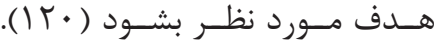

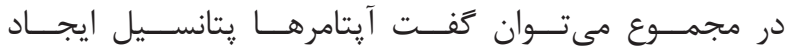

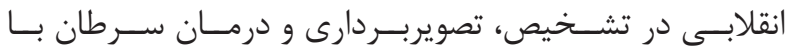

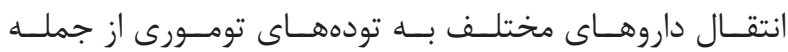

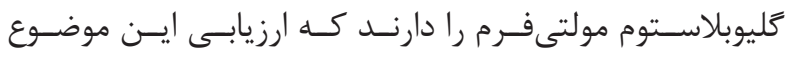

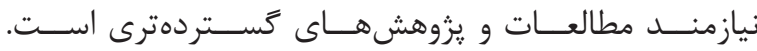

TEPP ,TENN

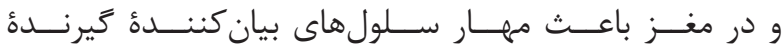

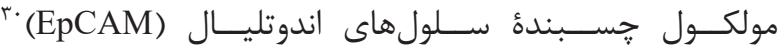

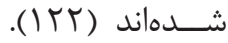

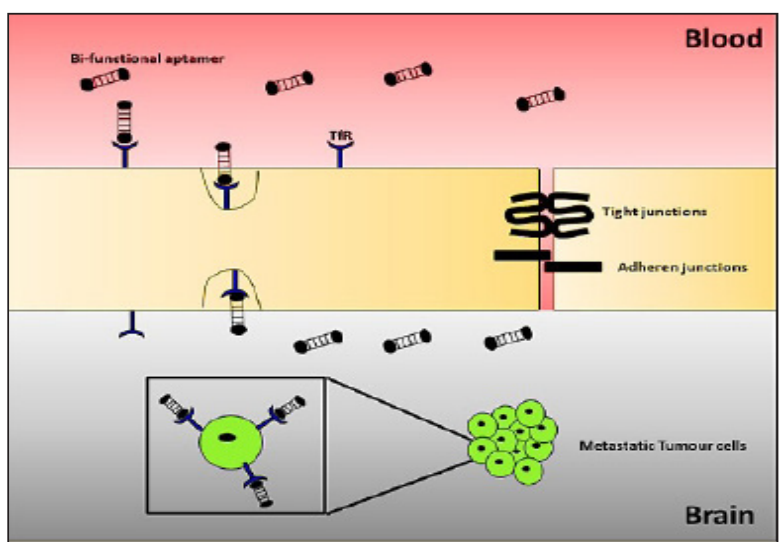

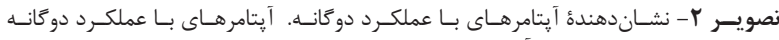

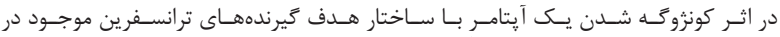

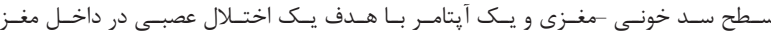

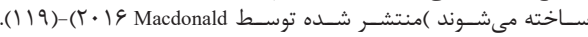

آيتامر هايیى با ساختار هاى هدف شناخته نشده از آنجايسى كـهـ روش Cell-SELEX از كل يـك سـلول بهعنوان

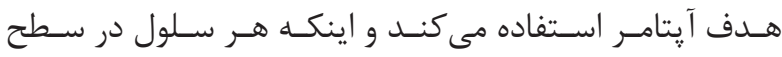

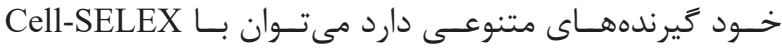

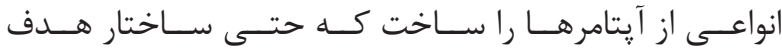

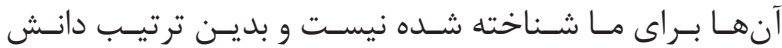

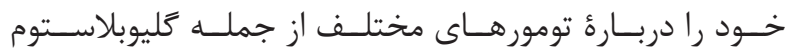

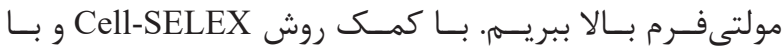

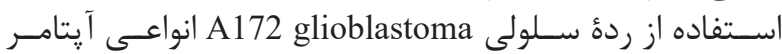

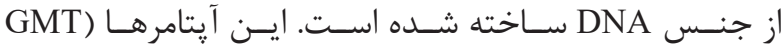

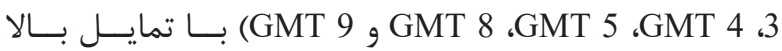

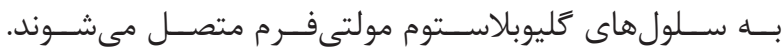
GMT و GMT 3

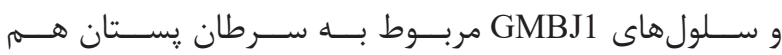

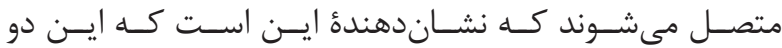

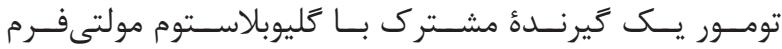

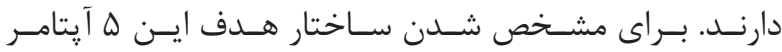

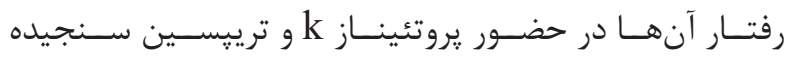

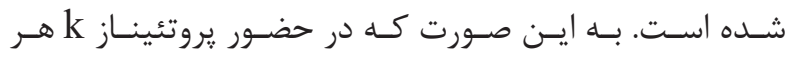

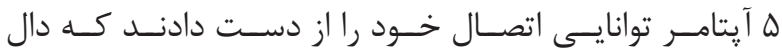

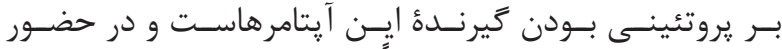

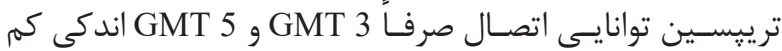

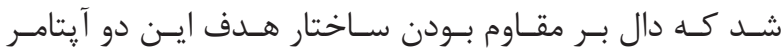

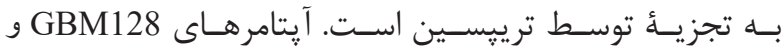

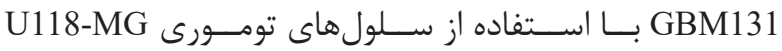


1. Louis DN, Perry A, Reifenberger G, Von Deimling A, Figarella-Branger D, Cavenee WK, et al. The 2016 world health organization classification of tumors of the central nervous system: a summary. Acta Neuropathologica. 2016; 131(6): 803-20.

2. Cerchia L, Esposito CL, Camorani S, Rienzo A, Stasio L, Insabato L, et al. Targeting Axl with an highaffinity inhibitory aptamer. Molecular Therapy. 2012; 20(12): 2291-303.

3. Ohgaki H, Kleihues P. Genetic pathways to primary and secondary glioblastoma. Am J Pathol. 2007; 170(5): 1445-53.

4. Appin CL, Brat DJ. Molecular pathways in gliomagenesis and their relevance to neuropathologic diagnosis. Adv Anat Pathol. 2015; 22(1): 50-8.

5. Dimitrov L, Hong CS, Yang C, Zhuang Z, Heiss JD. New developments in the pathogenesis and therapeutic targeting of the IDH1 mutation in glioma. Int J Med Sci. 2015; 12(3): 201-13.

6. Bayat N, Ebrahimi-Barough S, Norouzi-Javidan A, Saberi H, Tajerian R, Ardakan MMM, et al. Apoptotic effect of atorvastatin in glioblastoma spheroids tumor cultured in fibrin gel. Biomed Pharmacother. 2016; 84: 1959-66.

7. Cloughesy TF, Cavenee WK, Mischel PS. Glioblastoma: from molecular pathology to targeted treatment. Annu Rev Pathol. 2014; 9: 1-25.

8. Gilbert CA, Ross AH. Glioma stem cells: cell culture, markers and targets for new combination therapies. J Cell Biochem. 2009; 108(5): 1031-8.

9. Friedman HS, Prados MD, Wen PY, Mikkelsen T, Schiff D, Abrey LE, et al. Bevacizumab alone and in combination with irinotecan in recurrent glioblastoma. $\mathrm{J}$ Clin Oncol. 2009; 27(28): 4733-40.

10. Stupp R, Mason WP, Van Den Bent MJ, Weller M, Fisher B, Taphoorn MJ, et al. Radiotherapy plus concomitant and adjuvant temozolomide for glioblastoma. N Engl J Med. 2005; 352(10): 987-96.

11. Jakola AS, Gulati S, Weber C, Unsgård G, Solheim O. Postoperative deterioration in health related quality of life as predictor for survival in patients with glioblastoma: a prospective study. PLoS One. 2011; 6(12): e28592.

12. Thomas RP, Recht L, Nagpal S. Advances in the management of glioblastoma: the role of temozolomide and MGMT testing. Clin Pharmacol. 2013; 5: 1-9.
13. Omuro A, DeAngelis LM. Glioblastoma and other malignant gliomas: a clinical review. Jama. 2013; 310(17): 1842-50.

14. Capietto A-H, Keirallah S, Gross E, Dauguet N, Laprevotte E, Jean C, et al. Emerging concepts for the treatment of hematological malignancies with therapeutic monoclonal antibodies. Current drug Targets. 2010; 11(7): 790-800.

15. Gyorki DE, Spillane J, Speakman D, Shackleton M, Henderson MA. Current management of advanced melanoma: a transformed landscape. ANZ Journal of Surgery. 2014; 84(9): 612-7.

16. Camorani S, Cerchia L. Oligonucleotide aptamers for glioma targeting: an update. Central Nervous System Agents in Medicinal Chemistry (Formerly Current Medicinal Chemistry-Central Nervous System Agents). 2015; 15(2): 126-37.

17. Keefe AD, Pai S, Ellington A. Aptamers as therapeutics. Nature Reviews Drug Discovery. 2010; 9(7): $\quad 537-50$.

18. Thiel KW, Giangrande PH. Therapeutic applications of DNA and RNA aptamers. Oligonucleotides. 2009; 19(3): 209-22.

19. Cerchia L, De Franciscis V. Targeting cancer cells with nucleic acid aptamers. Trends Biotechnol. 2010; 28(10): 517-25.

20. de Franciscis V, Esposito CL, Catuogno S, Cellai L, Cerchia L. Aptamers as innovative diagnostic and therapeutic agents in the central nervous system. CNS Neurol Disord Drug Targets. 2009; 8(5): 393-401.

21. Ireson CR, Kelland LR. Discovery and development of anticancer aptamers. Mol Cancer Ther. 2006; 5(12): 2957-62.

22. Wu X, Zhao Z, Bai H, Fu T, Yang C, Hu X, et al. DNA aptamer selected against pancreatic ductal adenocarcinoma for in vivo imaging and clinical tissue recognition. Theranostics. 2015; 5(9): 985-94.

23. Li X, An Y, Jin J, Zhu Z, Hao L, Liu L, et al. Evolution of DNA aptamers through in vitro metastatic-cell-based systematic evolution of ligands by exponential enrichment for metastatic cancer recognition and imaging. Anal Chem. 2015; 87(9): 4941-8.

24. Li X, Zhang W, Liu L, Zhu Z, Ouyang G, An Y, et al. In vitro selection of DNA aptamers for metastatic breast cancer cell recognition and tissue imaging. Anal Chem. 
2014; 86(13): 6596-603.

25. Sefah K, Tang Z, Shangguan D, Chen H, LopezColon D, Li Y, et al. Molecular recognition of acute myeloid leukemia using aptamers. Leukemia. 2009; 23(2): 235-44.

26. Shangguan D, Meng L, Cao ZC, Xiao Z, Fang X, Li $\mathrm{Y}$, et al. Identification of liver cancer-specific aptamers using whole live cells. Anal Chem. 2008; 80(3): 721-8.

27. Wu Q, Wu L, Wang Y, Zhu Z, Song Y, Tan Y, et al. Evolution of DNA aptamers for malignant brain tumor gliosarcoma cell recognition and clinical tissue imaging. Biosens Bioelectron. 2016; 80: 1-8.

28. Tuerk C, Gold L. Systematic evolution of ligands by exponential enrichment: RNA ligands to bacteriophage T4 DNA polymerase. Science. 1990; 249(4968): 505-10.

29. Ellington AD, Szostak JW. In vitro selection of RNA molecules that bind specific ligands. Nature. 1990; 346(6287): 818-22.

30. Missailidis S, Hardy A. Aptamers as inhibitors of target proteins. Expert Opin Ther Pat. 2009; 19(8): 1073-82.

31. Ni X, Castanares M, Mukherjee A, Lupold SE. Nucleic acid aptamers: clinical applications and promising new horizons. Curr Med Chem. 2011; 18(27): 4206-14.

32. Ilgu M, Nilsen-Hamilton M. Aptamers in analytics. Analyst. 2016; 141(5): 1551-68.

33. Šmuc T, Ahn I-Y, Ulrich H. Nucleic acid aptamers as high affinity ligands in biotechnology and biosensorics. J Pharm Biomed Anal. 2013; 81: 210-7.

34. Keefe AD, Cload ST. SELEX with modified nucleotides. Curr Opin Chem Biol. 2008; 12(4): 448-56.

35. Chelliserrykattil J, Ellington AD. Evolution of a T7 RNA polymerase variant that transcribes 2'-O-methyl RNA. Nat Biotechnol. 2004; 22(9): 1155-60.

36. Burmeister PE, Lewis SD, Silva RF, Preiss JR, Horwitz LR, Pendergrast PS, et al. Direct in vitro selection of a 2'-O-methyl aptamer to VEGF. Chem Biol. 2005; 12(1): 25-33.

37. de Franciscis V, Rienzo A, Cerchia L. Nucleic acid aptamers for in vivo molecular imaging. Molecular Imaging: InTech; 2012.

38. Blind M, Blank M. Aptamer selection technology and recent advances. Mol Ther Nucleic Acids. 2015; 4(1): e223. doi: 10.1038/mtna.2014.74.
39. Le TT, Scott S, Cass AE. Streptavidin binding bifunctional aptamers and their interaction with low molecular weight ligands. Anal Chim Acta. 2013; 761: 143-8.

40. Bates PJ, Laber DA, Miller DM, Thomas SD, Trent JO. Discovery and development of the G-rich oligonucleotide AS1411 as a novel treatment for cancer. Exp Mol Pathol. 2009; 86(3): 151-64.

41. Soundararajan S, Wang L, Sridharan V, Chen W, Courtenay-Luck N, Jones D, et al. Plasma membrane nucleolin is a receptor for the anticancer aptamer AS1411 in MV4-11 leukemia cells. Mol Pharmacol. 2009; 76(5): 984-91.

42. Soundararajan S, Chen W, Spicer EK, CourtenayLuck N, Fernandes DJ. The nucleolin targeting aptamer AS1411 destabilizes Bcl-2 messenger RNA in human breast cancer cells. Cancer Res. 2008; 68(7): 2358-65.

43. Ko HY, Lee JH, Kang H, Ryu SH, Song IC, Lee DS, et al. A nucleolin-targeted multimodal nanoparticle imaging probe for tracking cancer cells using an aptamer. J Nucl Med. 2010; 51(1): 98-105.

44. Rosenberg JE, Bambury RM, Van Allen EM, Drabkin HA, Lara PN, Harzstark AL, et al. A phase II trial of AS1411 (a novel nucleolin-targeted DNA aptamer) in metastatic renal cell carcinoma. Invest New Drugs. 2014; 32(1): 178-87.

45. Hicke BJ, Marion C, Chang Y-F, Gould T, Lynott $\mathrm{CK}$, Parma D, et al. Tenascin-C aptamers are generated using tumor cells and purified protein. J Biol Chem. 2001; 276(52): 48644-54.

46. Erickson HP, Bourdon MA. Tenascin: an extracellular matrix protein prominent in specialized embryonic tissues and tumors. Annu Rev Cell Biol. 1989; 5(1): 71-92.

47. Tiitta O, Virtanen I, Sipponen P, Gould V. Tenascin expression in inflammatory, dysplastic and neoplastic lesions of the human stomach. Virchows Archiv. 1994; 425(4): 369-74.

48. Herold-Mende C, Mueller MM, Bonsanto MM, Schmitt HP, Kunze S, Steiner HH. Clinical impact and functional aspects of tenascin-C expression during glioma progression. Int J Cancer. 2002; 98(3): 362-9.

49. Leins A, Riva P, Lindstedt R, Davidoff MS, Mehraein $\mathrm{P}$, Weis $\mathrm{S}$. Expression of tenascin-C in various human brain tumors and its relevance for survival in patients with astrocytoma. Cancer. 2003; 98(11): 2430-9.

50. Daniels DA, Chen H, Hicke BJ, Swiderek KM, Gold L. A tenascin-C aptamer identified by tumor cell SELEX: systematic evolution of ligands by exponential 


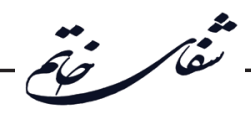

enrichment. Proc Natl Acad Sci U S A. 2003; 100(26): 15416-21.

51. Chen X-C, Deng Y-L, Lin Y, Pang D-W, Qing H, $\mathrm{Qu}$ F, et al. Quantum dot-labeled aptamer nanoprobes specifically targeting glioma cells. Nanotechnology. 2008; 19(23): 235105. doi: 10.1088/0957$4484 / 19 / 23 / 235105$

52. Li Y, Qiao H, Yan W, Zhang J, Xing C, Wang H, et al. Molecular recognition force spectroscopy study of the dynamic interaction between aptamer GBI-10 and extracellular matrix protein tenascin- $\mathrm{C}$ on human glioblastoma cell. J Mol Recognit. 2013; 26(1): 46-50.

53. Jacobson O, Yan X, Niu G, Weiss ID, Ma Y, Szajek LP, et al. PET imaging of tenascin-C with a radiolabeled single-stranded DNA aptamer. J Nucl Med. 2015; 56(4): 616-21.

54. Li K, Deng J, Jin H, Yang X, Fan X, Li L, et al. Chemical modification improves the stability of the DNA aptamer GBI-10 and its affinity towards tenascin-C. Organic \& Biomolecular Chemistry. 2017; 15(5): 1174-82.

55. Gan HK, Cvrljevic AN, Johns TG. The epidermal growth factor receptor variant III (EGFRvIII): where wild things are altered. FEBS J. 2013; 280(21): 5350-70.

56. Li B, Yuan M, Kim I-A, Chang C-M, Bernhard EJ, Shu H-KG. Mutant epidermal growth factor receptor displays increased signaling through the phosphatidylinositol-3 kinase/AKT pathway and promotes radioresistance in cells of astrocytic origin. Oncogene. 2004; 23(26): 4594-602.

57. Wu X, Liang H, Tan Y, Yuan C, Li S, Li X, et al. Cell-SELEX aptamer for highly specific radionuclide molecular imaging of glioblastoma in vivo. PloS One. 2014; 9(3): e90752.

58. Tan Y, Shi Y-s, Wu X-d, Liang H-y, Gao Y-b, Li S-j, et al. DNA aptamers that target human glioblastoma multiforme cells overexpressing epidermal growth factor receptor variant III in vitro. Acta Pharmacol Sin. 2013; 34(12): 1491-8.

59. Zhang X, Liang H, Tan Y, Wu X, Li S, Shi Y. A U87-EGFRvIII cell-specific aptamer mediates small interfering RNA delivery. Biomed Rep. 2014; 2(4): 495-9.

60. Tang J, Huang N, Zhang X, Zhou T, Tan Y, Pi J, et al. Aptamer-conjugated PEGylated quantum dots targeting epidermal growth factor receptor variant III for fluorescence imaging of glioma. Int J Nanomedicine 2017; 12: 3899-3911.
61. Esposito CL, Passaro D, Longobardo I, Condorelli G, Marotta P, Affuso A, et al. A neutralizing RNA aptamer against EGFR causes selective apoptotic cell death. PLoS One. 2011; 6(9): e24071.

62. Camorani S, Crescenzi E, Colecchia D, Carpentieri A, Amoresano A, Fedele M, et al. Aptamer targeting EGFRvIII mutant hampers its constitutive autophosphorylation and affects migration, invasion and proliferation of glioblastoma cells. Oncotarget. 2015; 6(35): 37570-87.

63. Padfield E, Ellis HP, Kurian KM. Current therapeutic advances targeting EGFR and EGFRvIII in glioblastoma. Front Oncol. 2015; 5: doi: 10.3389/fonc.2015.00005.

64. Li N, Nguyen HH, Byrom M, Ellington AD. Inhibition of cell proliferation by an anti-EGFR aptamer. PloS One. 2011; 6(6): e20299.

65. Wan Y, Mahmood MAI, Li N, Allen PB, Kim Yt, Bachoo R, et al. Nanotextured substrates with immobilized aptamers for cancer cell isolation and cytology. Cancer. 2012; 118(4): 1145-54.

66. Wan Y, Liu Y, Allen PB, Asghar W, Mahmood MAI, Tan J, et al. Capture, isolation and release of cancer cells with aptamer-functionalized glass bead array. Lab Chip. 2012; 12(22): 4693-701.

67. Liu Z, Han L, Dong Y, Tan Y, Li Y, Zhao M, et al. EGFRvIII/integrin $\beta 3$ interaction in hypoxic and vitronectinenriching microenvironment promote GBM progression and metastasis. Oncotarget. 2016; 7(4): 4680-94.

68. Cerchia L, Esposito CL, Jacobs AH, Tavitian B, De Franciscis V. Differential SELEX in human glioma cell lines. PloS One. 2009; 4(11): e7971.

69. Stommel JM, Kimmelman AC, Ying H, Nabioullin R, Ponugoti AH, Wiedemeyer R, et al. Coactivation of receptor tyrosine kinases affects the response of tumor cells to targeted therapies. Science. 2007; 318(5848): 287-90.

70. Cerchia L, De Franciscis V. Nucleic acid aptamers against protein kinases. Curr Med Chem. 2011; 18(27): 4152-8.

71. Shinh Y-S, Lai C-Y, Kao Y-R, Shiah S-G, Chu Y-W, Lee $\mathrm{H}-\mathrm{S}$, et al. Expression of axl in lung adenocarcinoma and correlation with tumor progression. Neoplasia. 2005; 7(12): 1058-64.

72. Zhang Y-X, Knyazev PG, Cheburkin YV, Sharma $\mathrm{K}$, Knyazev YP, Örfi L, et al. AXL is a potential target for therapeutic intervention in breast cancer progression. 
Cancer Research. 2008; 68(6): 1905-15.

73. Cerchia L, Martinez Montero J-C, Monfared P. Signal transduction alterations in glioma: implications for diagnosis and therapy. J Signal Transduct. 2012; 2012. doi: $10.1155 / 2012 / 704247$.

74. Hutterer M, Knyazev P, Abate A, Reschke M, Maier $\mathrm{H}$, Stefanova N, et al. Axl and growth arrest-specific gene 6 are frequently overexpressed in human gliomas and predict poor prognosis in patients with glioblastoma multiforme. Clin Cancer Res. 2008; 14(1): 130-8.

75. Esposito CL, Nuzzo S, Kumar SA, Rienzo A, Lawrence CL, Pallini R, et al. A combined microRNAbased targeted therapeutic approach to eradicate glioblas toma stem-like cells. J Control Release. 2016; 238: 43-57.

76. Östman A. PDGF receptors-mediators of autocrine tumor growth and regulators of tumor vasculature and stroma. Cytokine Growth Factor Rev. 2004; 15(4): 275-86.

77. Gilbertson RJ, Clifford SC. PDGFRB is overexpressed in metastatic medulloblastoma. Nat Genet. 2003; 35(3): 197-8.

78. Camorani S, Esposito CL, Rienzo A, Catuogno $\mathrm{S}$, Iaboni M, Condorelli $\mathrm{G}$, et al. Inhibition of receptor signaling and of glioblastoma-derived tumor growth by a novel PDGFR $\beta$ aptamer. Mol Ther. 2014; 22(4): 828-41.

79. Yu D, Wang D, Zhu F-G, Bhagat L, Dai M, Kandimalla ER, et al. Modifications incorporated in $\mathrm{CpG}$ motifs of oligodeoxynucleotides lead to antagonistactivity of tolllike receptors 7 and 9. J Med Chem. 2009; 52(16): 510814.

80. Group ES. Preclinical and phase 1A clinical evaluation of an anti-VEGF pegylated aptamer (EYE001) for the treatment of exudative age-related macular degeneration. Retina. 2002; 22(2): 143-52.

81. Zhou J, Neff CP, Swiderski P, Li H, Smith DD, Aboellail $\mathrm{T}$, et al. Functional in vivo delivery of multiplexed anti-HIV-1 siRNAs via a chemically synthesized aptamer with a sticky bridge. Mol Ther. 2013; 21(1): 192-200.

82. Hays E, Duan W, Shigdar S. Aptamers and glioblastoma: their potential use for imaging and therapeutic applications. Int $\mathrm{J}$ Mol Sci. 2017; 18(12): 2576. doi: 10.3390/ijms18122576.

83. Singh SK, Hawkins C, Clarke ID, Squire JA, Bayani $\mathrm{J}$, Hide $\mathrm{T}$, et al. Identification of human brain tumour initiating cells. Nature. 2004; 432(7015): 396-401.
84. Brescia P, Ortensi B, Fornasari L, Levi D, Broggi G, Pelicci G. CD133 is essential for glioblastoma stem cell maintenance. Stem Cells. 2013; 31(5): 857-69.

85. Schmohl JU, Vallera DA. CD133, selectively targeting the root of cancer. Toxins (Basel). 2016; 8(6): 165. doi: 10.3390/toxins 8060165 .

86. Shigdar S, Qiao L, Zhou S-F, Xiang D, Wang T, Li Y, et al. RNA aptamers targeting cancer stem cell marker CD133. Cancer Lett. 2013; 330(1): 84-95.

87. Kim Y, Wu Q, Hamerlik P, Hitomi M, Sloan AE, Barnett GH, et al. Aptamer identification of brain tumor initiating cells. Cancer Res. 2013; 73(15): 4923-36.

88. Guo J, Gao X, Su L, Xia H, Gu G, Pang Z, et al. Aptamer-functionalized PEG-PLGA nanoparticles for enhanced anti-glioma drug delivery. Biomaterials. 2011; 32(31): 8010-20.

89. Shan L. Indotricarbocyanine-loaded AS1411 DNA aptamer-and TGN peptide-modified poly (ethylene glycol)-poly ( $\varepsilon$-caprolactone) nanoparticles. 2012.

90. Farokhzad OC, Cheng J, Teply BA, Sherifi I, Jon $\mathrm{S}$, Kantoff PW, et al. Targeted nanoparticle-aptamer bioconjugates for cancer chemotherapy in vivo. Proc Natl Acad Sci U S A. 2006; 103(16): 6315-20.

91. Bagalkot V, Farokhzad OC, Langer R, Jon S. An aptamer-doxorubicin physical conjugate as a novel targeted drug-delivery platform. Angew Chem Int Ed Engl. 2006; 45(48): 8149-52.

92. Chen H, Zheng X, Di B, Wang D, Zhang Y, Xia H, et al. Aptamer modification improves the adenoviral transduction of malignant glioma cells. J Biotechnol. 2013; 168(4): 362-6.

93. Thiel KW, Hernandez LI, Dassie JP, Thiel WH, Liu $\mathrm{X}$, Stockdale KR, et al. Delivery of chemo-sensitizing siRNAs to HER2+-breast cancer cells using RNA aptamers. Nucleic Acids Res. 2012; 40(13): 6319-37.

94. Zhou J, Rossi JJ. Aptamer-targeted cell-specific RNA interference. Silence. 2010; 1(1): 4. doi: 10.1186/1758907X-1-4.

95. Wu X, Ding B, Gao J, Wang H, Fan W, Wang X, et al. Second-generation aptamer-conjugated PSMAtargeted delivery system for prostate cancer therapy. Int J Nanomedicine. 2011; 6:1747-56.

96. Dai F, Zhang Y, Zhu X, Shan N, Chen Y. Anticancer role of MUC1 aptamer-miR-29b chimera in epithelial ovarian carcinoma cells through regulation of PTEN 


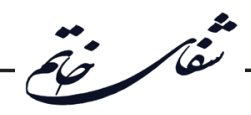

methylation. Target Oncol. 2012; 7(4): 217-25.

97. Liu N, Zhou C, Zhao J, Chen Y. Reversal of paclitaxel resistance in epithelial ovarian carcinoma cells by a MUC1 aptamer-let-7i chimera. Cancer Invest. 2012; 30(8): 577-82.

98. Esposito CL, Cerchia L, Catuogno S, De Vita G, Dassie JP, Santamaria G, et al. Multifunctional aptamermiRNA conjugates for targeted cancer therapy. Mol Ther. 2014; 22(6): 1151-63.

99. Das M, Duan W, Sahoo SK. Multifunctional nanoparticle-EpCAM aptamer bioconjugates: a paradigm for targeted drug delivery and imaging in cancer therapy. Nanomedicine. 2015; 11(2): 379-89.

100. Tian J, Ding L, Ju H, Yang Y, Li X, Shen Z, et al. A multifunctional nanomicelle for real-time targeted imaging and precise near-infrared cancer therapy. Angew Chem Int Ed Engl. 2014; 53(36): 9544-9.

101. Zhang Y, Zhang T, Liu M, Kuang Y, Zu G, Zhang $\mathrm{K}$, et al. Aptamer-targeted magnetic resonance imaging contrast agents and their applications. J Nanosci Nanotechnol. 2018; 18(6): 3759-74.

102. Da Pieve C, Perkins AC, Missailidis S. Anti-MUC1 aptamers: radiolabelling with $99 \mathrm{mTc}$ and biodistribution in MCF-7 tumour-bearing mice. Nucl Med Biol. 2009; 36(6): 703-10.

103. Gao H, Qian J, Yang Z, Pang Z, Xi Z, Cao S, et al. Whole-cell SELEX aptamer-functionalised poly (ethyleneglycol)-poly ( $\varepsilon$-caprolactone) nanoparticles for enhanced targeted glioblastoma therapy. Biomaterials. 2012; 33(26): 6264-72.

104. Hicke BJ, Stephens AW, Gould T, Chang Y, Lynott CK, Heil J, et al. Tumor targeting by an aptamer. J Nucl Med. 2006; 47(4): 668-78.

105. Jokerst JV, Lobovkina T, Zare RN, Gambhir SS. Nanoparticle PEGylation for imaging and therapy. Nanomedicine. 2011; 6(4): 715-28.

106. Ma H, Gao Z, Yu P, Shen S, Liu Y, Xu B. A dual functional fluorescent probe for glioma imaging mediated by blood-brain barrier penetration and glioma cell targeting. Biochem Biophys Res Commun. 2014; 449(1): 44-8.

107. Liu W, Fu Y, Xu S, Ding F, Zhao G, Zhang K, et al. c-Met expression is associated with time to recurrence in patients with glioblastoma multiforme. J Clin Neurosci. 2011; 18(1): 119-21.

108. Huang PH, Mukasa A, Bonavia R, Flynn RA,
Brewer ZE, Cavenee WK, et al. Quantitative analysis of EGFRvIII cellular signaling networks reveals a combinatorial therapeutic strategy for glioblastoma. Proc Natl Acad Sci U S A. 2007; 104(31): 12867-72.

109. Delač M, Motaln H, Ulrich H, Lah TT. Aptamer for imaging and therapeutic targeting of brain tumor glioblastoma. Cytometry A. 2015; 87(9): 806-16.

110. Patel MM, Goyal BR, Bhadada SV, Bhatt JS, Amin AF. Getting into the brain. CNS Drugs. 2009; 23(1): 35-58.

111. Baseri B, Choi JJ, Tung Y-S, Konofagou EE. Multi-modality safety assessment of blood-brain barrier opening using focused ultrasound and definity microbubbles: a short-term study. Ultrasound Med Biol. 2010; 36(9): 1445-59.

112. Calias P, Banks WA, Begley D, Scarpa M, Dickson P. Intrathecal delivery of protein therapeutics to the brain: a critical reassessment. Pharmacol Ther. 2014; 144(2): 114-22.

113. Bleier BS, Kohman RE, Feldman RE, Ramanlal S, Han X. Permeabilization of the blood-brain barrier via mucosal engrafting: implications for drug delivery to the brain. PLoS One. 2013; 8(4): e61694.

114. Sheikov N, McDannold N, Vykhodtseva N, Jolesz F, Hynynen K. Cellular mechanisms of the blood-brain barrier opening induced by ultrasound in presence of microbubbles. Ultrasound Med Biol. 2004; 30(7): 979-89.

115. Miyake MM, Bleier BS. The blood-brain barrier and nasal drug delivery to the central nervous system. Am J Rhinol Allergy. 2015; 29(2): 124-7.

116. Aprili D, Bandschapp O, Rochlitz C, Urwyler A, Ruppen W. Serious complications associated with external intrathecal catheters used in cancer pain patientsa systematic review and metaanalysis. Anesthesiology. 2009; 111(6): 1346-55.

117. Yu YJ, Zhang Y, Kenrick M, Hoyte K, Luk W, Lu Y, et al. Boosting brain uptake of a therapeutic antibody by reducing its affinity for a transcytosis target. Sci Transl Med. 2011; 3(84): 84ra44-84ra44.

118. Pestourie C, Tavitian B, Duconge F. Aptamers against extracellular targets for in vivo applications. Biochimie. 2005; 87(9-10): 921-30.

119. Macdonald J, Henri J, Goodman L, Xiang D, Duan W, Shigdar S. Development of a bifunctional aptamer targeting the transferrin receptor and epithelial cell adhesion molecule (EpCAM) for the treatment of brain cancer metastases. ACS Chem Neurosci. 2017; 8(4): 
777-84.

120. Eichler AF, Chung E, Kodack DP, Loeffler JS, Fukumura D, Jain RK. The biology of brain metastasestranslation to new therapies.Nat Rev Clin Oncol. 2011; 8(6): 344-56.

121. Ponka P, Lok CN. The transferrin receptor: role in health and disease. Int J Biochem Cell Biol. 1999; 31(10): 1111-37.

122. Bayrac AT, Sefah K, Parekh P, Bayrac C, Gulbakan
$\mathrm{B}$, Oktem HA, et al. In vitro selection of DNA aptamers to glioblastoma multiforme. ACS Chem Neurosci. 2011; 2(3): $175-81$.

123. Kang D, Wang J, Zhang W, Song Y, Li X, Zou Y, et al. Selection of DNA aptamers against glioblastoma cells with high affinity and specificity. PLoS One. 2012; 7(10): e42731.

124. Krex D, Klink B, Hartmann C, von Deimling A, Pietsch T, Simon M, et al. Long-term survival with glioblastoma multiforme. Brain. 2007; 130(10): 2596606. 